\title{
CONTAGIO DE LAS CRISIS FINANCIERAS INTERNACIONALES: EL CASO DE MÉXICO
}

\author{
Santiago Bazdresch \\ Alejandro M. Werner ${ }^{1}$
}

Noviembre de 2000

Documento de Investigación No. 2000-02

Dirección General de Investigación Económica

BANCO DE MÉXICO

1 Documento preparado para la conferencia del Banco Mundial: "Contagion: How It Spreads and How It Can Be Stopped", Washington, D.C.. Febrero 3 y 4 de 2000. Las opiniones contenidas en este documento representan las de los autores y no necesariamente las del Banco de México. Los autores agradecen los valiosos comentarios de Guillermo Perry y Soledad Martínez Peria sobre este trabajo. 


\title{
CONTAGIO DE LAS CRISIS FINANCIERAS INTERNACIONALES: EL CASO DE MÉXICO
}

\author{
Santiago Bazdresch
}

Alejandro M. Werner

Noviembre de 2000

Documento de Investigación No. 2000-02

\section{RESUMEN}

En este documento se estudia el contagio que sufrieron los mercados financieros en México durante las crisis en Asia, en Rusia y en Brasil en el periodo de 1997 - 1999. Se determina la magnitud del contagio a los mercados mexicanos utilizando diferentes métodos estadísticos. Posteriormente, se estudian los posibles canales de contagio hacia la economía mexicana, y se discute cómo la economía ha hecho frente a estos choques. 


\section{CONTAGIO DE LAS CRISIS FINANCIERAS INTERNACIONALES: EL CASO DE MÉXICO}

\section{Introducción}

México fue el epicentro de la crisis del tequila en 1994 y 1995 en la cual muchas de las características novedosas de las crisis recientes en los mercados emergentes hicieron su aparición. En particular, el tema de la presencia de contagio cautivó la atención de varios autores (por ejemplo, Valdés (1997), Sachs, Tornell y Velasco (1995) y Calvo y Reinhart (1996)). Desde entonces, las crisis de Asia, Rusia y Brasil han motivado numerosos documentos, sugiriendo que la investigación sobre el tema del contagio también puede ser contagiosa.

Afortunadamente, durante las últimas crisis en los mercados emergentes, México se ha mantenido en la periferia y apenas ha sufrido ligeramente los efectos del contagio. En este documento se estudia cómo la economía mexicana fue afectada por las crisis recientes y se describen las acciones de política implementadas para minimizar sus repercusiones.

La crisis financiera global impactó a las economías de América Latina principalmente a través de dos canales. Primero, el colapso de la producción en las economías asiáticas incidió sobre los precios mundiales de las mercancías, socavando los ingresos por exportaciones así como las finanzas públicas en algunos países de América Latina. Además, aquellas economías Latinoamericanas que mantienen vínculos comerciales cercanos con Asia, o que compiten con exportaciones asiáticas en otros mercados, sufrieron los efectos negativos de la baja en la demanda en Asia y de la depreciación de las monedas asiáticas. En el caso de México, en 1998 la economía experimentó un importante deterioro de sus términos de intercambio (5.5\%), principalmente debido a la caída en los precios del petróleo. Asimismo, la volatilidad en los mercados financieros

internacionales causó una reducción en la oferta de capital extranjero a la región, dando lugar a movimientos en los tipos de cambio y en las tasas de interés, generando un importante ajuste en la absorción interna.

Aun cuando el canal comercial fue el primer vehículo del contagio de la crisis asiática a Latinoamérica, el canal financiero fue seguramente el más importante. En particular, el colapso de la economía rusa, y la moratoria unilateral declarada sobre sus obligaciones internas y externas, resultaron ser dañinas para los mercados financieros internacionales. La consecuencia más importante fue que estos fenómenos indujeron un importante cambio cualitativo en la manera en que el riesgo soberano de las economías emergentes es evaluado por los participantes de los mercados. El hecho de que Rusia no recibiera ayuda institucional efectiva, hizo que el falso sentimiento de seguridad sobre las inversiones en mercados emergentes desapareciera, llevando a una revaluación sustancial de la probabilidad de moratoria en otras economías emergentes. Esta 
situación resultó en una recomposición de las carteras de activos de los inversionistas institucionales, desencadenando así un proceso de desinversión en las economías emergentes, a favor de las economías desarrolladas.

En este periodo, la percepción general del mercado de que la situación en Brasil era insostenible amplificó el contagio de la crisis rusa hacia América Latina. Por tanto, el proceso de liquidación de las posiciones en Brasil, llevó a la intensificación del contagio en otros países de la región. La anticipación de la devaluación brasileña hizo que sus efectos se sintieran antes de que ésta se diera. Así, cuando la devaluación finalmente ocurrió el 13 de enero de 1999, sus efectos sobre los mercados financieros fueron menores que lo que originalmente se anticipaba.

\section{Gráfica 1}

\section{Diferenciales (Spreads) de la deuda soberana en América Latina}

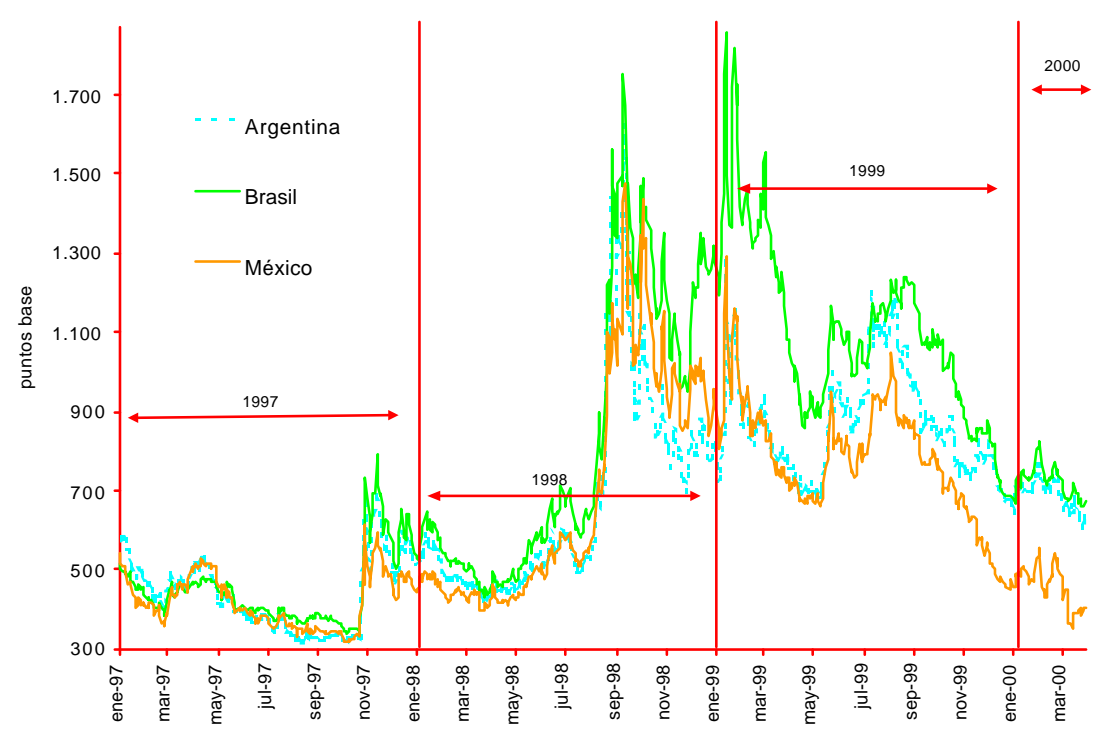

El entorno externo desfavorable orilló a varios países Latinoamericanos a endurecer sus políticas fiscal y monetaria. Por ejemplo, el gobierno de México encaró este entorno externo tomando una actitud de cautela extrema, dada la volatilidad de las expectativas de los inversionistas. Una de las lecciones de la crisis de 1994-1995 fue que, una vez que los mercados sobrereaccionan, la política económica también tiene que sobrereaccionar para restaurar la confianza. Siguiendo esta receta, la adecuación de las políticas fiscal y monetaria se estructuró sobre la base de que las perturbaciones a las que se enfrentaba la economía serían permanentes, aun cuando, como se observó posteriormente, existían elementos para justificar que representaban fenómenos de índole temporal.

A pesar de la crisis, la economía mexicana mostró un comportamiento favorable durante 1998, más aún si éste se compara con el de otras economías, tanto desarrolladas como emergentes. Lo anterior fue resultado de una respuesta adecuada de política, y de que la amplia integración con la economía 
de los Estados Unidos que ha tenido lugar durante la última década, le ha permitido a la economía mexicana beneficiarse de la expansión económica que ha tenido lugar en Estados Unidos en los últimos cinco años.

El resto del documento se estructura de la siguiente manera: en la sección 2 se determina la magnitud del contagio de las crisis asiática, rusa y brasileña a la economía mexicana. En la sección 3 se discuten los posibles canales de contagio de estas crisis así como las medidas implementadas por las autoridades mexicanas. Finalmente, en la última sección, se presentan algunas conclusiones.

\section{Medición del Contagio}

En esta sección se utilizan distintos métodos estadísticos para estimar la magnitud del contagio sufrido por los mercados financieros de México a causa de la crisis financiera internacional que tuvo lugar en el periodo 1997-1999. Con este propósito, se utilizan los trabajos realizados por Baig y Goldfajn (1999) y por Rigobón (1999). En primer lugar, se calculan coeficientes de correlación y se estiman vectores autorregresivos (VAR's) para medir el grado de comovimiento entre los distintos mercados mexicanos y los correspondientes mercados en los países en crisis. También se examina si estas correlaciones mostraron un aumento significativo durante los periodos de crisis utilizando varios métodos: la prueba t heteroscedástica de dos muestras desarrollada por Forbes y Rigobón (1999), regresiones de cambio de régimen de Hamilton, y finalmente, la prueba de especificación desarrollada por Rigobón (1999). Las correlaciones, por su naturaleza contemporánea, son una medida muy intuitiva de contagio, representado exactamente lo que uno se imagina en primera instancia al pensar sobre este tema. Además de la información contenida en las correlaciones, se utilizan VARs, los cuales permiten observar respuestas rezagadas, medir el tiempo que tardan las perturbaciones en desaparecer y proveen una primera aproximación al problema de causalidad. Finalmente, las tres pruebas estadísticas sobre los estimadores de correlación, aportan argumentos más sólidos para aceptar o rechazar la existencia de contagio.

El periodo de observación se extiende del primero de enero de 1996 al 10 de junio de 1999. El año de 1996 representa un buen periodo de control ya que en ese entonces los mercados se habían recuperado de la crisis de 1994-1995, y aún no comenzaban las otras crisis. La crisis asiática se inicia el dos de julio de 1997, fecha de la devaluación del baht tailandés; la crisis rusa a partir del 17 de agosto de 1998, con la declaración de la moratoria de pagos; y la crisis brasileña el 13 de enero de 1999, cuando el real inició su transición a un régimen de tipo de cambio flotante. Para la estimación de los VARs se distingue entre los distintos países asiáticos, en cuyo caso se toma la crisis de Malasia que comenzó el 14 de julio de 1997; la crisis en Indonesia a partir del 14 de agosto; la crisis de Corea el 6 de noviembre del mismo año, y la crisis en Filipinas a partir del 11 de julio, también del mismo año. Se utilizan asimismo datos de Singapur, tomando como fecha de inicio de la crisis en ese país el 12 de julio. Finalmente, para esclarecer en mayor medida la crisis asiática se considera una segunda ronda que comienza con el ataque especulativo a la bolsa de valores de Hong Kong y al dólar de este país hacia el final de 1997. En los últimos meses de 1997 
los mercados accionarios y cambiarios de los países de la región, incluido Japón, sufrieron un segundo golpe. El won coreano y el yen japonés se depreciaron $40 \%$ y $10 \%$ respectivamente entre octubre y diciembre de 1997. En esas fechas Indonesia sufrió varios ataques, Korea pidió ayuda al FMI y el mercado accionario Hang-Seng de Hong Kong se desplomó. A estos eventos se les denomina en el presente trabajo la "segunda ola" de la crisis asiática. Desde el punto de vista de otros mercados emergentes, fue después de estos sucesos que hubo un cambio sustancial en la percepción de la crisis asiática como un peligro sistémico, y que el contagio a otras economías tuvo más alcance. Durante este tiempo, los tipos de cambio de las monedas de Brasil y Rusia fueron atacados por inversionistas extranjeros, quienes observaban desequilibrios considerados insostenibles, en cada uno de estos países. Sin embargo, ambos países lograron mantener su esquema cambiario por algunos meses más.

Para medir el contagio en los distintos mercados se utiliza la información que se escribe a continuación. Para los mercados accionarios se usan los valores diarios del cierre de los principales índices de cada país: el Bovespa para Brasil, el Kospi para Corea, el JCI para Indonesia, el Klci para Malasia, el IPC para México, el Pcomp para Filipinas, el Aspmt para Rusia, el Sti para Singapur y el Set para Tailandia. Para los diferenciales de deuda soberana se toman los índices EMBI de los países para los que están disponibles y para los que no se cuenta con este indicador se construyen como en Baig y Goldfajn (1999). Los tipos de cambio también son los valores de cierre de cada moneda expresados en unidades de la moneda nacional por dólares americanos.

\section{II.1 Correlación entre mercados y significancia de los aumentos en correlación}

La medida más intuitiva de contagio es el aumento en la correlación entre los mercados de México y los de otros países. Por tanto, se entiende por contagio, aquellos aumentos en los coeficientes de correlación entre los mercados mexicanos y los del país donde se originó la crisis que tuvieron lugar durante el periodo de crisis.

Con este propósito, para cada crisis se construyen estimadores de correlación durante cuatro periodos distintos para mostrar un panorama relativamente completo de los eventos. Un intervalo de tiempo muy corto, de dos días antes de la crisis hasta una semana después; uno intermedio, de dos semanas antes de la crisis hasta tres meses después de su comienzo; un segundo periodo de tres meses, empezando tres meses después de que la crisis surgió; y finalmente, un intervalo de seis meses, comenzando dos semanas antes del principio de la crisis. Se espera que el coeficiente de correlación de corto plazo dé cuenta de grandes comovimientos de una sola vez, y que los intervalos de mediano y largo plazo ayuden a explicar la persistencia de los incrementos en correlación. Los periodos de cálculo empiezan un poco antes de que las crisis se declaren oficialmente. Ello con el propósito de incluir un periodo en el que aunque los mercados están siendo atacados, y tal vez se comporten como en una crisis, esta crisis aún no es generalizada. Por otra parte, al escoger las fechas de esta forma se reduce la importancia sobre los resultados de las fechas especificas utilizadas para identificar el principio de las crisis. 
En estos ejercicios se encuentra fuerte evidencia de contagio en los mercados de deuda soberana y en las bolsas de valores después de la segunda ola de la crisis asiática. En el caso de los tipos de cambio, el contagio está presente, pero no de una manera tan clara como en los otros dos casos. Además, la validez de estos últimos resultados es cuestionable si se toma en cuenta el régimen de tipo de cambio que prevalecía en estas economías antes de las devaluaciones. A continuación se presentan los resultados para cada mercado.

\section{Mercado cambiario}

Aparentemente durante una crisis financiera si existe una relación distinta del peso mexicano con los movimientos de otras monedas. Se observa evidencia de contagio inmediato durante la crisis asiática y más aún durante la segunda ola de esta crisis. También se puede ver algo de contagio, hacia el peso, del rublo ruso al principio de la crisis rusa, y del real brasileño después de la devaluación del 13 de enero de 1999. Estos resultados, sin embargo, no son robustos, pues antes de la crisis la mayoría de estas monedas estaba en un régimen de tipo de cambio fijo o en una banda cambiaria. Los coeficientes de correlación muestran evidencia de que las crisis financieras internacionales han incrementado la correlación de los mercados mexicanos con los de otros países emergentes, así como indicios de contagio directo de los países en crisis hacia México.

Se utiliza una prueba t heteroscedástica de dos muestras ${ }^{2}$ con el fin de evaluar la significancia estadística de estos resultados. En el cuadro 1 se reporta cualquier incremento de varianza entre el periodo tranquilo y el periodo de crisis con significancia estadística mayor que $1 \%(* * *), 5 \%(* *)$ o $10 \%(*)$.

2 Una descripción detallada de esta prueba se presenta en Rigobón (1999). 
Cuadro 1

Coeficientes de correlación entre tipos de cambio para distintos periodos

\begin{tabular}{|c|c|c|c|c|c|c|c|c|c|}
\hline & & BRASIL & RUSIA & COREA & INDONESIA & MALASIA & FILIPINAS & SINGAPUR & TAILANDIA \\
\hline \multicolumn{2}{|c|}{ Periodo tranquilo } & 0.00 & 0.04 & -0.03 & 0.03 & 0.04 & -0.01 & 0.03 & -0.01 \\
\hline \multirow{4}{*}{ 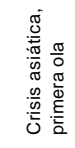 } & Primera semana & -0.47 & -0.52 & -0.45 & $0.41^{\star \star *}$ & $0.38^{\text {** }}$ & 0.01 & -0.13 & $0.81^{* * *}$ \\
\hline & Primeros tres meses & -0.10 & -0.06 & $0.05^{* * *}$ & -0.11 & -0.10 & $0.09 \star \star \star *$ & -0.10 & $0.18^{\star \star *}$ \\
\hline & Segundos tres meses & $0.16^{* * *}$ & 0.01 & $0.12^{* * *}$ & $0.16^{\star \star \star}$ & -0.04 & 0.01 & $0.10^{* * *}$ & -0.04 \\
\hline & Primeros seis meses & $0.12^{* * *}$ & 0.00 & $0.12^{\star \star *}$ & 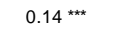 & -0.05 & 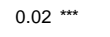 & $0.06^{* * *}$ & $0.02^{\star \star *}$ \\
\hline \multirow{4}{*}{ 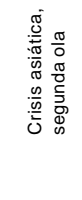 } & Primeros tres meses & $0.26^{* * *}$ & -0.12 & $0.13^{\star \star *}$ & $0.21^{\star * *}$ & 0.02 & 0.04 & $0.14^{* * *}$ & $0.15^{* \star *}$ \\
\hline & Segundos tres meses & $0.22^{\star * *}$ & 0.00 & $0.34^{\star * *}$ & 0.02 & $0.19^{* * *}$ & -0.01 & $0.19^{* * *}$ & $0.14^{\star \star *}$ \\
\hline & Primeros seis meses & $0.25^{\star * *}$ & -0.09 & $0.15^{\star * *}$ & $0.15^{\star \star \star}$ & $0.07^{* * *}$ & 0.02 & $0.16^{* * *}$ & $0.15^{* \star *}$ \\
\hline & Primera semana & -0.09 & -0.41 & 0.15 & $0.37^{\star \star}$ & 0.29 * & 0.15 & 0.21 & -0.14 \\
\hline \multirow{4}{*}{ 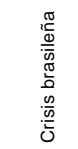 } & Primera semana & -0.08 & -0.52 & 0.07 & $0.38^{* *}$ & 0.08 & $0.31^{* *}$ & $0.53^{* * *}$ & $0.30^{* *}$ \\
\hline & Primeros tres meses & $0.15^{\star * *}$ & -0.28 & $0.11^{* * *}$ & $0.24^{\star \star \star}$ & 0.04 & 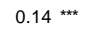 & 0.00 & $0.20^{\star * *}$ \\
\hline & Segundos tres meses & $0.16^{* * *}$ & -0.10 & $0.01 * *$ & $0.33^{\star \star \star}$ & -0.13 & 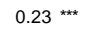 & 0.01 & $0.20^{* \star *}$ \\
\hline & Primeros seis meses & $0.14^{\star \star \star}$ & -0.25 & $0.08^{\star \star *}$ & $0.26^{\star \star \star}$ & 0.02 & 0.00 & 0.00 & $0.19^{* \star *}$ \\
\hline
\end{tabular}

En el caso de Brasil, se puede apreciar un incremento significativo del coeficiente de correlación a lo largo del periodo de seis meses posterior a la devaluación del real. No obstante, sorprendentemente para una depreciación de esa magnitud, el incremento en este coeficiente no es significativo de inmediato. En cuanto a Asia, se observa un incremento generalizado de la correlación con México, durante los seis meses posteriores al principio de la crisis en Tailandia, de mercados que casi no exhiben correlación alguna en el periodo tranquilo, particularmente los de Indonesia y Corea. Sin embargo, es en la segunda ola de la crisis asiática donde se observa el mayor contagio de las crisis en estos países. Nuevamente, estos incrementos en los coeficientes de correlación tomaron un tiempo en aparecer y los coeficientes de la primera semana son negativos. También hay señales de contagio de la crisis rusa, pero desaparecen rápidamente.

La segunda ola de la crisis asiática, así como la crisis rusa llevaron a un aumento generalizado en la volatilidad. Es notorio que el contagio importante a México de los países de Asia se materializó sólo después de los eventos del cuarto trimestre de 1997, probablemente como reflejo de cambios drásticos en la percepción sobre las perspectivas de las economías de los mercados emergentes. Se encuentran altas correlaciones, hasta de 0.26 con Brasil (aún con un régimen de banda cambiaria), 0.32 con Rusia, 0.21 con Indonesia y 0.34 con Corea. Cabe señalar que todas estas correlaciones habían sido prácticamente iguales a cero durante el periodo tranquilo. Es importante recordar que estos países mantenían un régimen de bandas de tipo de cambio antes de que las crisis ocurrieran, limitando cualquier posible correlación. En consecuencia, los incrementos en correlación tal vez sólo indiquen la relación latente entre estas variables, que había sido oscurecida por los regímenes vigentes. 


\section{Mercados accionarios}

Los incrementos en las correlaciones entre los mercados accionarios son un fenómeno mucho más generalizado, especialmente hacia al interior de regiones geográficas. A lo largo del periodo de muestra, la correlación entre el IPC de México y el BOVESPA de Brasil nunca es menor que 0.27. La crisis de Asia provocó un aumento en la correlación del mercado accionario mexicano con los mercados de los países en crisis, particularmente durante la segunda ola de dicha crisis. La correlación con los países asiáticos se eleva durante esos meses, de -0.04 a 0.21 con Corea, de 0.04 a 0.39 con las Filipinas y de 0.03 a 0.40 con Singapur. Esta relación entre los mercados accionarios de Asia y de México es, desde la perspectiva de las correlaciones, el caso más sobresaliente de contagio. Por su parte, la crisis rusa también aumentó la correlación del mercado mexicano con el ruso, ya que esta correlación es significativamente mayor que la observada en el periodo tranquilo, aunque este fenómeno se presentó desde el principio de la crisis asiática. Si se compara con el periodo tranquilo, la crisis brasileña si provocó un aumento de la correlación entre los índices accionarios de México y de Brasil, aunque dicha correlación no es tan grande como durante la crisis en Rusia.

\section{Cuadro 2}

Coeficientes de correlación de mercados accionarios durante distintos periodos

\begin{tabular}{|c|c|c|c|c|c|c|c|c|c|}
\hline & & BRASIL & RUSIA & COREA & INDONESIA & MALASIA & FILIPINAS & SINGAPUR & TAILANDIA \\
\hline \multicolumn{2}{|c|}{ Periodo tranquilo } & 0.27 & -0.03 & -0.01 & 0.23 & 0.14 & 0.11 & 0.17 & 0.13 \\
\hline \multirow{4}{*}{ 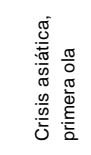 } & Primera semana & 0.35 & -0.25 & -0.28 & -0.20 & -0.15 & 0.24 & $0.46^{\star \star}$ & -0.27 \\
\hline & Primeros tres meses & $0.47^{* * *}$ & $0.30^{* \star *}$ & -0.02 & $0.31^{\star \star \star}$ & 0.09 & $0.21^{* \star *}$ & $0.28^{\star \star \star *}$ & 0.07 \\
\hline & Segundos tres meses & $0.79^{* * \star}$ & $0.08^{* \star \star}$ & $0.18^{* \star *}$ & -0.03 & -0.01 & -0.11 & 0.01 & -0.15 \\
\hline & Primeros seis meses & $0.68^{\star \star *}$ & $0.12^{\star \star \star}$ & $0.17^{\star \star \star}$ & 0.04 & 0.02 & -0.01 & 0.08 & -0.05 \\
\hline \multirow{5}{*}{ 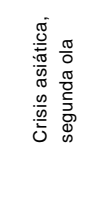 } & Primera semana & $0.54^{\star *}$ & -0.76 & -0.56 & -0.40 & -0.62 & -0.54 & 0.01 & -0.62 \\
\hline & Primeros tres meses & $0.65^{* * *}$ & $0.26^{* * *}$ & $0.17^{\star \star \star}$ & 0.25 & $0.26^{* * *}$ & $0.35^{* * *}$ & $0.37^{\star \star \star *}$ & $0.20^{\star \star \star}$ \\
\hline & Segundos tres meses & $0.64^{* \star *}$ & $0.33^{* * *}$ & $0.34^{\star \star \star}$ & $0.31^{\star \star \star}$ & $0.39^{* * *}$ & $0.17^{* * *}$ & $0.38^{\star \star \star *}$ & $0.39^{\star \star \star}$ \\
\hline & Primeros seis meses & $0.65^{\star \star *}$ & $0.29^{* \star *}$ & $0.21^{\star \star *}$ & $0.26^{\star \star \star}$ & $0.29^{\star \star \star}$ & $0.30^{* * *}$ & $0.36^{\star \star \star}$ & $0.26^{\star \star \star}$ \\
\hline & Primera semana & $0.80^{\star \star \star}$ & -0.22 & -0.69 & 0.25 & 0.12 & -0.16 & -0.17 & 0.11 \\
\hline \multirow{3}{*}{ 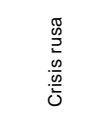 } & Primeros tres meses & $0.81^{\star * \star}$ & $0.13^{\star \star \star}$ & 0.00 & -0.03 & 0.14 & $0.22^{\star \star \star *}$ & $0.25^{\star \star \star}$ & 0.07 \\
\hline & Segundos tres meses & $0.63^{\star * \star}$ & $0.24^{* \star \star}$ & 0.00 & 0.15 & 0.05 & 0.04 & $0.20^{* \star}$ & 0.16 * \\
\hline & Primeros seis meses & $0.73^{\star * *}$ & $0.16^{* * *}$ & 0.00 & 0.02 & 0.12 & $0.17^{* * *}$ & $0.23^{\star \star *}$ & 0.10 \\
\hline \multirow{4}{*}{ 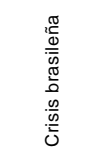 } & Primera semana & $0.85^{\star \star \star}$ & 0.22 * & -0.47 & 0.27 & $0.45^{\star \star}$ & -0.07 & $0.59^{\star \star \star}$ & 0.31 \\
\hline & Primeros tres meses & $0.65^{\star \star \star}$ & $0.20^{\star \star \star}$ & -0.05 & 0.04 & 0.05 & -0.06 & $0.26^{\star \star *}$ & 0.03 \\
\hline & Segundos tres meses & $0.68^{\star \star *}$ & $0.10^{* * *}$ & 0.02 * & $0.27^{\star \star \star}$ & -0.03 & $0.23^{* * *}$ & -0.14 & 0.05 \\
\hline & Primeros seis meses & $0.65^{* * *}$ & $0.15^{* \star \star}$ & -0.02 & 0.09 & 0.01 & 0.02 & 0.13 & 0.02 \\
\hline
\end{tabular}

La correlación del mercado mexicano con los mercados de otros países, particularmente Brasil, durante la crisis rusa, es significativamente distinta de la del periodo tranquilo. Después de la crisis rusa, muy probablemente los participantes en los mercados anticiparon la crisis de Brasil, por lo que se dio un ataque sostenido contra el real, aunque Brasil logró sobrellevar esta situación por unos meses más. Lo anterior llevó a una alta correlación entre los mercados mexicanos y brasileños 
después de la crisis en Rusia. De forma similar, fue durante la segunda ola de la crisis asiática cuando se produjo la reacción más fuerte de los participantes del mercado, incrementándose significativamente todas las medidas de correlación de la muestra. La alta correlación con los mercados asiáticos desaparece tras las crisis de estos países y, durante los episodios de crisis de Brasil y Rusia, se incrementa en algunos casos, pero de manera transitoria.

\section{Mercado de deuda soberana}

Durante las crisis, los precios de la deuda soberana mexicana estuvieron más correlacionados con los de la deuda de los países en crisis que en el periodo tranquilo.

\section{Cuadro 3}

Coeficientes de correlación de diferenciales (spreads) de deuda soberana durante distintos periodos

\begin{tabular}{|c|c|c|c|c|c|c|c|c|}
\hline & & BRASIL & RUSIA & COREA & INDONESIA & MALASIA & FILIPINAS & TAILANDIA \\
\hline \multicolumn{2}{|c|}{ Periodo tranquilo } & 0.66 & -0.02 & -0.01 & 0.05 & -0.18 & 0.13 & NA \\
\hline \multirow{4}{*}{ 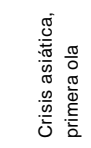 } & Primera semana & $0.87^{\star * *}$ & -0.29 & -0.52 & $0.36^{* \star}$ & -0.41 & $0.73^{\star \star \star *}$ & . \\
\hline & Primeros tres meses & $0.71^{* * *}$ & $0.06^{* \star \star}$ & -0.07 & 0.01 & $0.06^{* \star *}$ & $0.25^{* * *}$ & . \\
\hline & Segundos tres meses & $0.79^{\star \star \star}$ & $0.06^{* * \star}$ & $0.23^{\star \star \star}$ & $0.08^{*}$ & $0.47^{\star \star \star}$ & $0.62^{* \star *}$ & . \\
\hline & Primeros seis meses & $0.79^{\star * *}$ & $0.07^{* * \star}$ & $0.22^{* \star *}$ & $0.08^{\star \star \star}$ & $0.25^{\star \star *}$ & $0.59^{\star \star *}$ & . \\
\hline \multirow{5}{*}{ 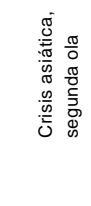 } & Primera semana & $0.91^{* * *}$ & 0.02 & 0.15 & -0.46 & $0.51^{\star \star \star *}$ & $0.88^{\star * *}$ & . \\
\hline & Primeros tres meses & $0.69^{\star \star \star}$ & $0.13^{\star \star \star}$ & $0.14^{\star \star \star}$ & 0.02 & $0.02^{\star \star \star}$ & $0.46^{\star \star \star}$ & . \\
\hline & Segundos tres meses & $0.85^{\star \star *}$ & $0.23^{* * \star}$ & $0.05^{\star \star \star}$ & $0.47^{\star \star \star}$ & $0.33^{\star \star \star}$ & $0.53^{\star \star \star *}$ & . \\
\hline & Primeros seis meses & $0.76^{* * *}$ & $0.17^{\star * \star}$ & $0.11^{\star \star \star *}$ & $0.17^{\star \star \star}$ & $0.04^{* * *}$ & $0.48^{\star \star *}$ & . \\
\hline & Primera semana & 0.50 & -0.14 & 0.00 & -0.05 & -0.07 & 0.26 & . \\
\hline \multirow{3}{*}{$\begin{array}{l}\frac{\pi}{0} \\
\frac{0}{2} \\
\frac{0}{0} \\
\frac{0}{0} \\
0\end{array}$} & Primeros tres meses & $0.82^{* * *}$ & $0.17^{* * \star}$ & $0.20^{\star \star \star}$ & $0.21^{\star \star \star}$ & $-0.01^{* * *}$ & $0.57^{\star \star *}$ & . \\
\hline & Segundos tres meses & $0.82^{* * *}$ & $0.20^{* \star \star}$ & $0.50^{\star \star \star *}$ & $0.27^{\star \star \star}$ & $0.17^{\star \star \star}$ & $0.60^{\star * *}$ & . \\
\hline & Primeros seis meses & $0.81^{\star * \star}$ & $0.18^{\star \star \star \star}$ & $0.26^{\star \star \star}$ & $0.20^{\star \star \star}$ & $0.02^{* * *}$ & $0.57^{\star \star \star}$ & . \\
\hline \multirow{4}{*}{ 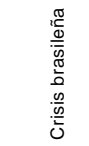 } & Primera semana & 0.72 & 0.25 ** & $0.76^{\star \star \star}$ & -0.35 & -0.46 & 0.17 & . \\
\hline & Primeros tres meses & $0.83^{\star \star \star}$ & $0.33^{\star \star \star *}$ & $0.56^{\star \star \star}$ & $0.25^{\star \star \star}$ & $0.14^{* \star *}$ & $0.59^{\star \star *}$ & . \\
\hline & Segundos tres meses & $1.00^{* \star *}$ & $0.98^{* * \star}$ & $0.99^{\star \star \star *}$ & $0.98^{\star \star \star}$ & $0.99^{\star \star \star}$ & $0.99^{\star \star \star}$ & . \\
\hline & Primeros seis meses & $0.98^{* * *}$ & $0.94^{* * *}$ & $0.95^{* \star \star}$ & $0.93^{* \star *}$ & $0.94^{* \star *}$ & $0.96^{* * *}$ & . \\
\hline
\end{tabular}

Durante los primeros tres meses de la crisis asiática, la correlación del precio de la deuda mexicana con la de los países asiáticos tuvo un incremento generalizado. La segunda ola de la crisis asiática y la moratoria de la deuda rusa, así como la devaluación de la moneda de este país produjeron los aumentos más notorios en estas correlaciones de spreads de deuda soberana. De nuevo, la correlación con Brasil no aumenta más después de la crisis de este país, evidencia de que la crisis de este país se anticipó antes de que sucediera y, por tanto, su efecto hacia el mercado mexicano se dio de manera anticipada. 


\section{II.2 Análisis de Vectores Autorregresivos (VAR)}

El análisis VAR tiene algunas ventajas sobre los simples coeficientes de correlación. Este análisis permite considerar efectos rezagados y contemporáneos de un mercado sobre otro, así como cuantificar la magnitud y significancia de la influencia de un mercado sobre otro. Para cada país, utilizamos datos diarios durante tres meses, empezando dos semanas antes de que la crisis de cada país comenzara. Este periodo de tiempo nos permite calcular si existen efectos de largo plazo o si las relaciones entre los mercados que se presentan inmediatamente después de la crisis son fenómenos transitorios o permanentes.

En cuanto a los tipos de cambio, se encuentran fuertes efectos sobre el mercado cambiario mexicano de la devaluación brasileña; en el caso de los mercados accionarios se encuentran efectos importantes de las crisis de Brasil y de algunos países asiáticos y en cuanto a los mercados de deuda soberana hay evidencia de un impacto significativo durante las crisis de Rusia, Brasil y la mayoría de los países asiáticos.

\section{Mercado cambiario}

De acuerdo con el análisis VAR el tipo de cambio no se vio directamente afectado por movimientos en el valor de otras monedas, excepto por el real brasileño. 


\section{Gráfica 2}

Respuesta del tipo de cambio del peso mexicano ante una innovación de una desviación estándar en el tipo de cambio de cada uno de los países durante sus crisis

Corea

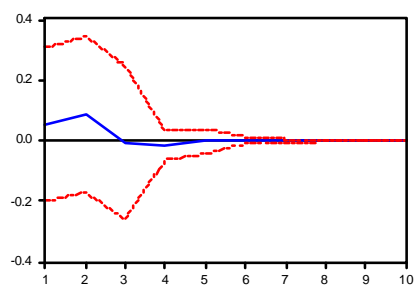

Filipinas

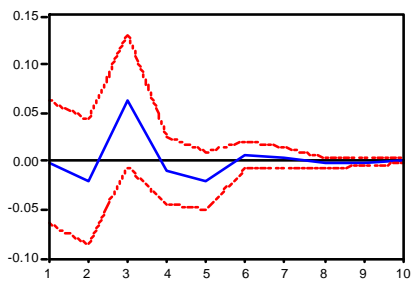

Indonesia

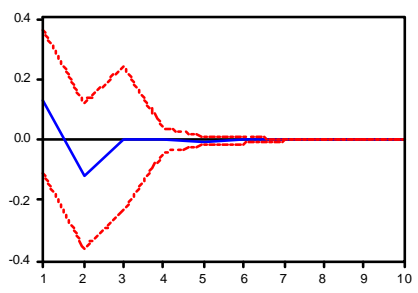

Singapur

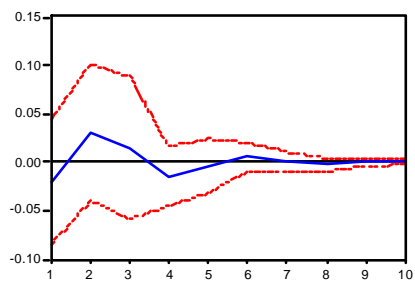

Malasia

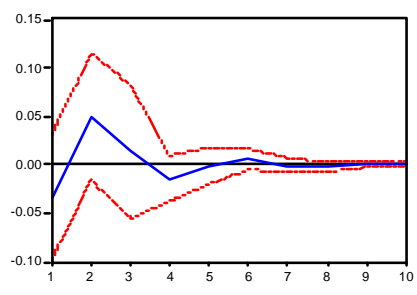

Tailandia

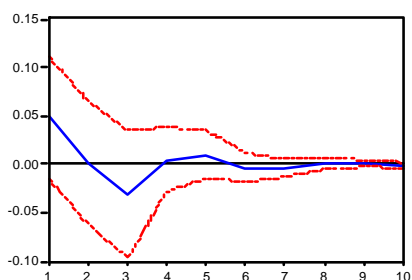

\section{Rusia}

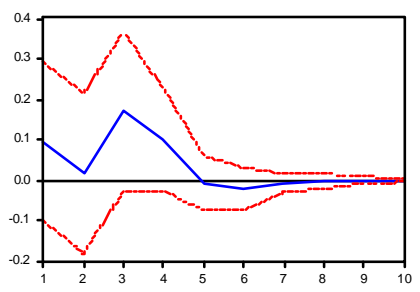

Brasil

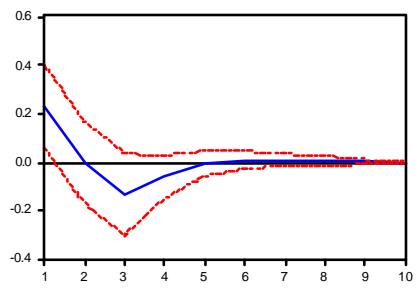

El tipo de cambio no sufrió impactos directos de los movimientos en el precio de las monedas asiáticas durante las crisis de estos países. En general, los efectos estimados tienen el signo correcto, pero no son significativos. Su respuesta a movimientos en el valor del rublo durante la crisis rusa tampoco resulta significativa en ningún momento. Por otra parte, hay una respuesta estadísticamente significativa del tipo de cambio ante movimientos en el valor del real brasileño frente al dólar. Como en el caso del análisis de correlaciones, los mercados cambiarios no parecen ser muy susceptibles al contagio, particularmente más allá de fronteras regionales.

\section{Mercados accionarios}

En cuanto a las bolsas de valores, se encuentra que durante la crisis asiática, los efectos de los movimientos en los mercados asiáticos no provocaron cambios muy significativos en la bolsa de valores de México. Los movimientos de los mercados de Corea, Malasia, y Tailandia no tienen 
ningún efecto importante, y los demás países tienen efectos poco relevantes. La respuesta a un choque proveniente de Indonesia es de mayor magnitud, aunque también es poco significativa.

\section{Gráfica 3}

Respuesta de la bolsa de valores de México a una innovación de una desviación estándar en las principales bolsas de valores de cada país durante sus crisis

Corea

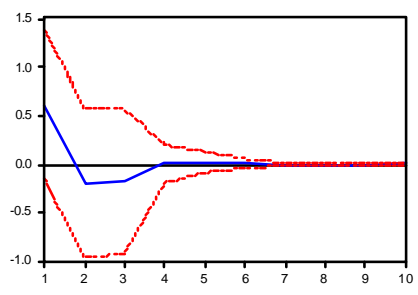

Filipinas

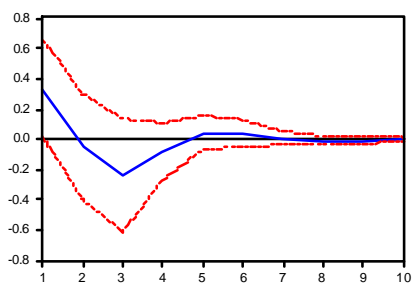

Indonesia

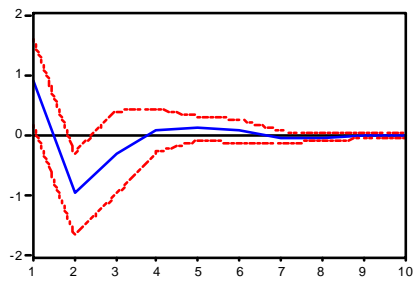

Singapur

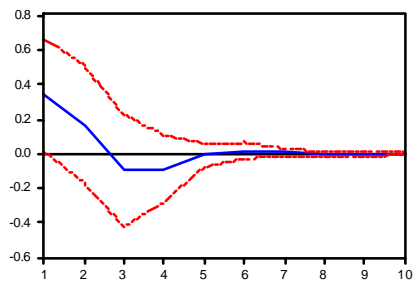

Malasia

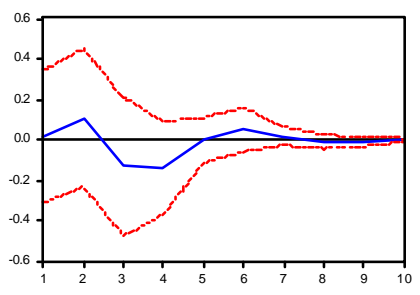

Tailandia

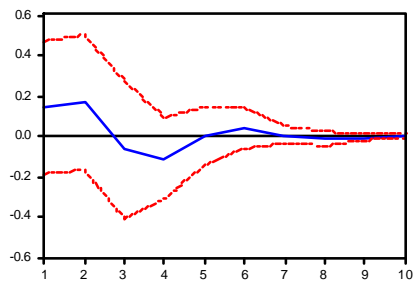

Rusia

Brasil
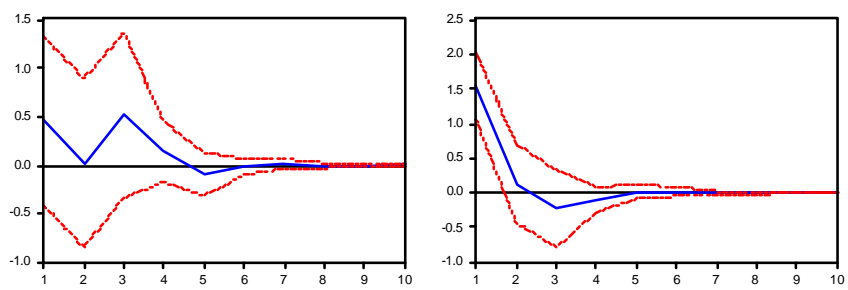

La respuesta a los movimientos del mercado ruso es similar. Por otra parte, durante la crisis de Brasil, el mercado accionario de este país tuvo cierta influencia sobre el mercado mexicano. Las reacciones a aquel tienen el signo correcto, son estadísticamente significativas y más aún, la magnitud de la respuesta es grande. Aun cuando no es claro si este movimiento es específico a los periodos de crisis, es claro que cualquier movimiento significativo de la bolsa de valores de Brasil se siente en los mercados mexicanos. 


\section{Diferenciales (Spreads) de deuda soberana}

Se estimaron VARs para los "spreads" de deuda soberana de México y de los países en crisis utilizando las variables en niveles en lugar de en diferencias. Lo anterior debido a que las pruebas de raíz unitaria indican que no hay evidencia de que estas series sean no estacionarias.

\section{Gráfica 4}

Respuesta de los diferenciales (spreads) de deuda de México a una innovación de una desviación estándar en el "spread" de la deuda de los países durante sus crisis

Corea

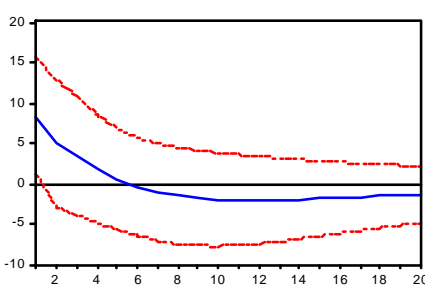

Indonesia

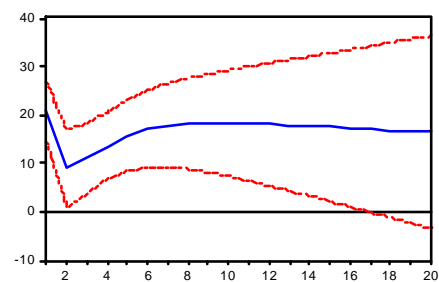

Malasia

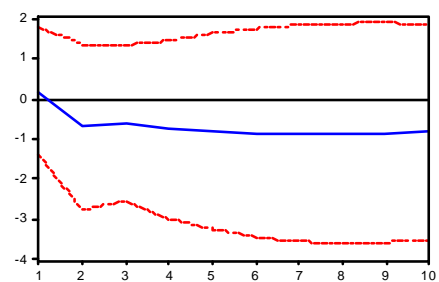

Filipinas

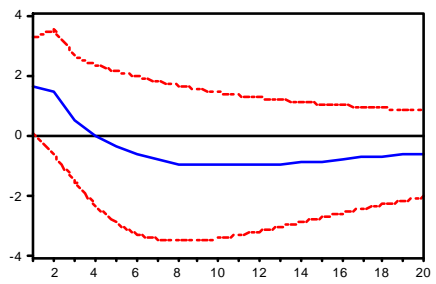

Rusia

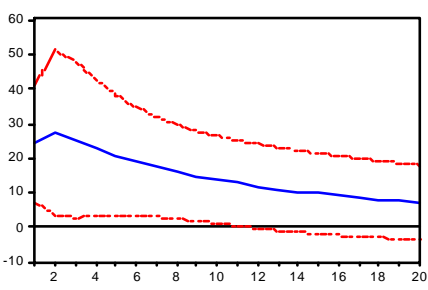

Tailandia

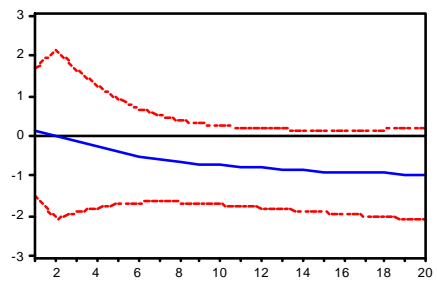

Brasil

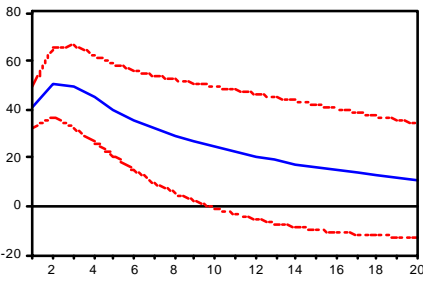

El efecto del "spread" de la deuda de los países asiáticos en México es reducido, pero duradero. Así, las reacciones a los aumentos en el precio de la deuda de Filipinas, Corea, Indonesia y Tailandia son significativos en algún punto, pero la reacción a Malasia no lo es. Como en el caso de los mercados de tipo de cambio y de valores, las reacciones a Rusia y a Brasil son más agudas. Un incremento en el "spread" soberano de la deuda rusa provocó un aumento importante en el "spread" de la deuda mexicana durante varios periodos. Con Brasil, el efecto sobre el mercado de deuda mexicana fue inmediato, significativo y también duradero. 


\section{Resumen del análisis VAR}

El efecto en los mercados mexicanos de los movimientos de los mercados de países en crisis se resume a continuación:

\begin{tabular}{|c|c|c|c|}
\cline { 2 - 4 } \multicolumn{1}{c|}{} & Tipo de cambio & Mercado de valores & Spreads soberanos \\
\hline Crisis asiática & $\begin{array}{c}\text { No hay efectos } \\
\text { significativos. }\end{array}$ & $\begin{array}{c}\text { Hay efectos } \\
\text { significativos de } \\
\text { Indonesia, Filipinas, y } \\
\text { Singapur. Respuesta } \\
\text { grande a Indonesia. }\end{array}$ & $\begin{array}{c}\text { Efectos significativos y } \\
\text { duraderos de algunos } \\
\text { países asiáticos. }\end{array}$ \\
\hline Crisis rusa & No hay efecto. & No hay efecto. & $\begin{array}{c}\text { Respuesta significativa y } \\
\text { duradera. }\end{array}$ \\
\hline Crisis brasileña & Reacción pequeña. & $\begin{array}{c}\text { Respuesta fuerte y } \\
\text { significativa de } \\
\text { magnitud importante. }\end{array}$ & $\begin{array}{c}\text { Respuesta grande, } \\
\text { significativa y duradera. }\end{array}$ \\
\hline
\end{tabular}

\section{II.3 Análisis de cambio de régimen}

En esta sección se utilizan las regresiones de cambio de régimen introducidas por Hamilton (1994), con el propósito de obtener evidencia de contagio. Se pretende explicar el comportamiento de los mercados mexicanos utilizando como variable explicativa los movimientos de los mercados de países en crisis. Se espera encontrar que durante los periodos tranquilos la influencia de otros mercados sobre los mercados nacionales es pequeña o despreciable, mientras que representan una variable explicativa importante durante tiempos de crisis. Se anticipa que los dos regímenes estimados estén estrechamente relacionados en alguna medida con la percepción acerca de un comportamiento diferente entre periodos de crisis y episodios tranquilos. Además que muestren evidencia de que durante tiempos de más volatilidad los coeficientes de las variables explicativas resulten más grandes que durante periodos tranquilos.

Con este fin, se construyó un modelo de cambio de régimen, donde se permite que los coeficientes de las variables explicativas, así como la varianza del error de estimación cambie entre un régimen y otro. La técnica de estimación consiste en optimizar la función de máxima verosimilitud, que supone un error con distribución normal en un modelo de la siguiente forma:

$$
y_{t}=\beta_{0}+\sum_{i=1}^{n} \beta_{i}^{j} x_{i}+\varepsilon_{i}^{j}
$$

donde $\boldsymbol{\beta}_{i}^{j}$ depende del régimen, $(\mathrm{j}=1,2)$ y el error $\boldsymbol{\varepsilon}_{t}^{j}$ es una variable con media cero y desviación estándar $\sigma^{j}$ la cual también depende del régimen. El procedimiento de estimación también genera estimadores de la probabilidad de que el proceso se encuentre en cada uno de los posibles regímenes. Se utilizan solamente las variables financieras de Rusia y Brasil como 
variables explicativas pues al introducir información de otros países se observa que no proveen información adicional.

\section{Cuadro 4}

Resultados de la estimación de cambio de régimen

\begin{tabular}{|c|c|c|c|c|c|c|c|c|}
\hline \multirow[t]{2}{*}{ Mercado } & & \multicolumn{3}{|c|}{ Régimen de crisis } & \multicolumn{3}{|c|}{ Régimen tranquilo } & \multirow[t]{2}{*}{ Log-verosimilitud } \\
\hline & & Coeficientes & Varianza & $\begin{array}{l}\text { Probabilidad } \\
\text { Ergódica }\end{array}$ & Coeficientes & Varianza & $\begin{array}{l}\text { Probabilidad } \\
\text { Ergódica }\end{array}$ & \\
\hline \multirow{2}{*}{$\begin{array}{l}\text { Tipo de } \\
\text { cambio }\end{array}$} & Brasil & $\begin{array}{c}0.36 \\
(0.22)\end{array}$ & \multirow{2}{*}{$\begin{array}{c}0.001 \\
(0.0001)\end{array}$} & \multirow[t]{2}{*}{0.16} & $\begin{array}{l}0.015 \\
(0.015)\end{array}$ & 0.00001 & \multirow[t]{2}{*}{0.84} & \multirow[t]{2}{*}{6622.4} \\
\hline & Russia & $\begin{array}{c}0.03 \\
(0.01)\end{array}$ & & & $\begin{array}{l}0.019 \\
(0.07)\end{array}$ & $(0.000001)$ & & \\
\hline \multirow{2}{*}{$\begin{array}{l}\text { Mercado } \\
\text { accionario }\end{array}$} & Brasil & $\begin{array}{c}0.39 \\
(0.038)\end{array}$ & \multirow{2}{*}{$\begin{array}{l}0.00063 \\
(0.0001)\end{array}$} & \multirow[t]{2}{*}{0.26} & $\begin{array}{l}0.025 \\
(0.034)\end{array}$ & 0.0001 & \multirow[t]{2}{*}{0.74} & \multirow[t]{2}{*}{5236.75 * } \\
\hline & Rusia & $\begin{array}{c}0.15 \\
(0.025)\end{array}$ & & & $\begin{array}{c}0.06 \\
(0.015)\end{array}$ & $(0.00001)$ & & \\
\hline \multirow{2}{*}{$\begin{array}{l}\text { "Spreads" } \\
\text { de deuda } \\
\text { soberana }\end{array}$} & Brasil & $\begin{array}{c}0.68 \\
(0.03)\end{array}$ & \multirow{2}{*}{$\begin{array}{c}0.00001 \\
(0.000002)\end{array}$} & \multirow[t]{2}{*}{0.39} & $\begin{array}{r}-0.005 \\
(0.02)\end{array}$ & \multirow{2}{*}{$\begin{array}{l}0.0000005 \\
(0.0000001)\end{array}$} & \multirow{2}{*}{0.61} & \multirow[t]{2}{*}{3438.46 * } \\
\hline & Rusia & $\begin{array}{c}0.56 \\
(0.08)\end{array}$ & & & $\begin{array}{l}-0.29 \\
(1.33)\end{array}$ & & & \\
\hline
\end{tabular}

Los valores en paréntesis son los estimadores del error estándar de los coeficientes.

* El logaritmo de la función de verosimilitud es significativamente más grande para la especificación de cambio de régimen que para la de un sólo régimen, al nivel de $10 \%$.

Los resultados muestran dos regímenes claramente identificados para cada uno de los mercados en consideración. Existe un régimen con poca varianza o "tranquilo", donde el proceso se ubica la mayor parte del tiempo y donde los resultados en los mercados de otros países tienen poca influencia sobre los mercados mexicanos. Asimismo, se observa un régimen de alta volatilidad o de crisis, en el que otros mercados alrededor del mundo son útiles como variables explicativas, y qué no se presenta con tanta frecuencia. El incremento en los coeficientes asociados a los mercados extranjeros, entre el periodo tranquilo y el periodo de crisis, es sustancial. Se encuentra que el movimiento del BOVESPA brasileño sólo tuvo un coeficiente de 0.025 durante el periodo tranquilo, y un coeficiente de 0.39 durante los tiempos de alta volatilidad o de crisis para explicar al mercado mexicano. Para los "spreads" de deuda soberana también se observan cambios notables en el coeficiente de Brasil. Así, éste es de 0.69 durante tiempos de crisis y de sólo 0.005 durante tiempos tranquilos.

Con el propósito de hacer pruebas de significancia, se estima el mismo modelo pero con coeficientes de la variable explicativa constantes, es decir, con el mismo coeficiente $\boldsymbol{\beta}_{i}^{j}$ en la ecuación (1) para ambos regímenes. Esto resulta en una significancia de 10\% para el cambio de régimen de los coeficientes, tanto en los "spreads" de deuda soberana cómo en el mercado accionario. De estas estimaciones se puede concluir que existe alguna evidencia de contagio de los mercados de Rusia y de Brasil hacia los mercados mexicanos. 
Las probabilidades "suavizadas"” del proceso de cambio de régimen estimado muestran la concordancia entre los periodos observados de crisis y aquéllos que el algoritmo de estimación identifica como periodos de crisis o de alta volatilidad. Las gráficas de las probabilidades "suavizadas" se muestran para los procesos del tipo de cambio y de los "spreads" de deuda soberana. El régimen de crisis, en el caso de los "spreads" de deuda coincide con la volatilidad en la valuación del riesgo soberano observada después de la declaración de moratoria de Rusia sobre su deuda. En el caso del tipo de cambio, el periodo de crisis refleja cortos periodos de devaluación y de alta volatilidad en los mercados internacionales.

Diferenciales (Spreads) soberanos de México y la probabilidad de régimen de crisis

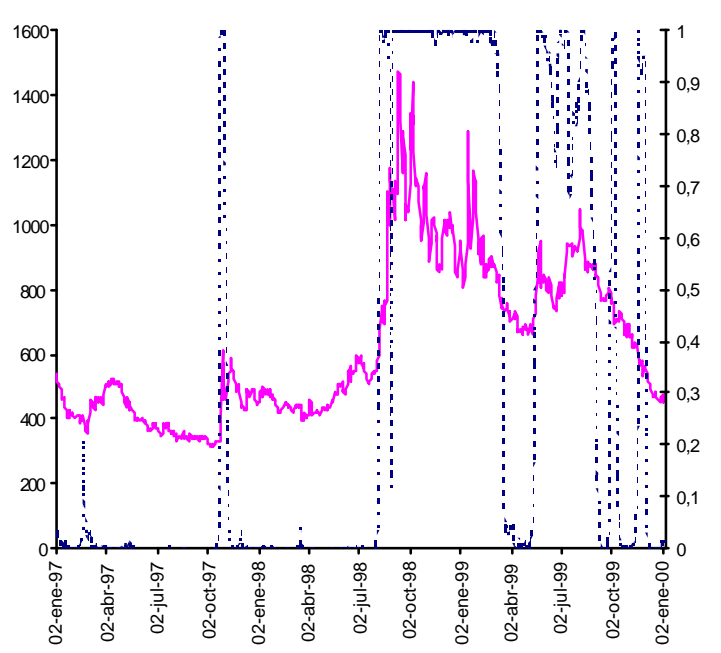

Tipo de cambio ya la probabilidad de régimen de crisis

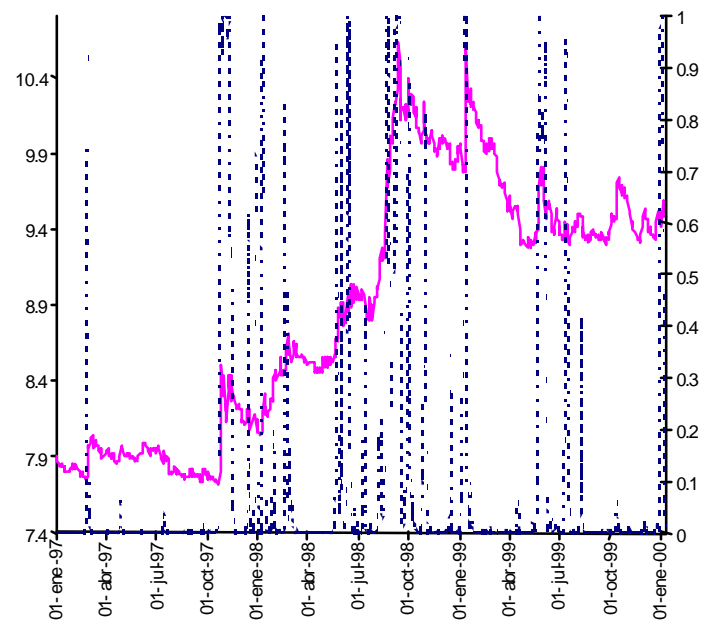

\section{II.4 Prueba de especificación}

Siguiendo el trabajo realizado por Rigobón (1999), en esta sección se utiliza un esquema alternativo para determinar la existencia de contagio. La idea es probar si los incrementos observados en la covarianza entre dos mercados se pueden atribuir a un cambio en la relación entre ellos, o solamente es el resultado de un incremento en la varianza de alguno de ellos, sin alterar el esquema de interrelación de los mercados.

Con este fin se construyen dos estimadores de variables instrumentales, para medir la relación entre un mercado foráneo y uno nacional, que serán iguales bajo la hipótesis de ausencia de cambio estructural. Posteriormente, se hace una prueba de significancia para detectar si existe alguna diferencia entre estos dos estimadores. Esto constituye una prueba de sobreidentificación, o una

3 Las probabilidades de transición entre estados se calculan en las regresiones de cambio de régimen, utilizando en cada periodo la información de los periodos anteriores. Sin embargo, el cálculo de las 
prueba de especificación de Hausmann, la cual permite establecer si la transmisión de perturbaciones es estable cuando la varianza de uno de los mercados aumenta.

El modelo en el que se sustenta dicha prueba es el siguiente:

$$
\begin{aligned}
& y_{t}=\beta x_{t}+\varepsilon_{t} \\
& x_{t}=\eta_{t},
\end{aligned}
$$

donde $x$ representa el mercado extranjero, $y$ representa el mercado nacional, y $\eta$ y $\varepsilon$ son perturbaciones estocásticas, con independencia entre $\eta$, y $\varepsilon$.

Rigobón argumenta que mientras las dos perturbaciones, $\eta$ y $\varepsilon$, no estén correlacionadas, la covarianza entre las dos variables se incrementará proporcionalmente a la varianza de la variable independiente $x$, mientras que la varianza de la variable dependiente aumentará menos que proporcionalmente. Esto a su vez provocará que cualquier incremento en la varianza de $x$, resulte en un aumento en el coeficiente de correlación, aún en ausencia de cambios estructurales.

Para resolver este problema el autor propone construir una prueba utilizando dos matrices de varianza covarianza. En un modelo completo de la forma siguiente,

$$
\begin{aligned}
& y_{t}=\beta x_{t}+\gamma z_{t}+\varepsilon_{t} \\
& x_{t}=\alpha y_{t}+z_{t}+\eta_{t}
\end{aligned}
$$

la resta de la matriz de covarianza entre $x$ y $y$ antes (l) y después (h) de un incremento en la varianza de $x, \sigma_{\eta}^{h}=(1+\delta) \sigma_{\eta}^{l}$, será igual a:

$$
\Delta \Omega_{t}=\Omega_{h}-\Omega_{l}=\frac{\delta \sigma_{\eta}^{2}}{(1-\alpha \beta)^{2}}\left(\begin{array}{cc}
\beta^{2} & \beta \\
\beta & 1
\end{array}\right)
$$

De aquí es claro que se pueden obtener dos estimadores independientes de , es decir:

$$
\begin{aligned}
& \hat{\beta}^{1}=\frac{\operatorname{COV}^{h}(x, y)-\operatorname{COV}^{l}(x, y)}{\operatorname{VAR}^{h}(x)-\operatorname{VAR}^{l}(x)} \\
& \hat{\beta}^{2}=\frac{\operatorname{VAR}^{h}(y)-\operatorname{VAR}^{l}(y)}{\operatorname{COV}^{h}(x, y)-\operatorname{COV}^{l}(x, y)}
\end{aligned}
$$

probabilidades "suavizadas" se realiza utilizando en cada periodo toda la información disponible, incluyendo la de los periodos sucesivos. 
o $\Delta \Omega_{12} / \Delta \Omega_{22}$ y $\Delta \Omega_{11} / \Delta \Omega_{12}$, donde $\operatorname{VAR}^{\mathrm{h}}$ y $\mathrm{COV}^{\mathrm{h}}$ se calculan con los datos del periodo de crisis, y $\mathrm{VAR}^{1}$ y $\mathrm{COV}^{1}$ se calculan con información del periodo tranquilo o de baja volatilidad.

Bajo la hipótesis de un aumento de $\sigma_{\eta}^{2}$ estos dos números serían estimadores de $\beta$, mientras que bajo la hipótesis de un aumento de $\sigma_{\varepsilon}^{2}$ o de $\sigma_{z}^{2}$ en lugar de uno de $\sigma_{\eta}^{2}$, estos dos números serían estimadores de $\alpha$ o de $\frac{\beta+\gamma}{1+\alpha \gamma}$ respectivamente. En cualquier caso, suponiendo un incremento de cualquiera de estas varianzas ambos coeficientes deben tener el mismo valor bajo la hipótesis de que no existe cambio estructural.

Los siguientes cuadros muestran ambos estimadores de $\beta$, para la relación entre un mercado nacional y uno extranjero. Al igual que en el trabajo de Rigobón, en muy pocos casos se encuentra evidencia significativa de cambios estructurales. En los cuadros se presentan los dos estimadores y, entre paréntesis, la estimación del error estándar de la diferencia entre ellos. Siguiendo la práctica utilizada en el resto del documento se utilizan periodos más largos tanto para los episodios de crisis y como para los tranquilos, que los que usa Rigobón. Se toma el año de 1996 como el periodo tranquilo, y para cada crisis, se utiliza una ventana de tres meses después de la fecha de inicio.

\section{Mercado accionario}

\section{Cuadro 5}

Resultados de la prueba de identificación para el mercado accionario

\begin{tabular}{|c|c|c|c|c|c|c|c|c|}
\hline & Brasil & Rusia & Corea & Indonesia & Malasia & Filipinas & Singapur & Tailandia \\
\hline & $\beta^{2}$ & $\beta^{2}$ & $\beta^{2}$ & $\beta^{2}$ & $\beta^{2}$ & $\beta^{2}$ & $\beta^{2}$ & $\beta^{2}$ \\
\hline $\begin{array}{c}\text { Crisis } \\
\text { asiática, } \\
\text { primera ola }\end{array}$ & $\begin{array}{c}0,24 \quad 0,35 \\
(6.95)\end{array}$ & $\begin{array}{c}-0,68 \quad 0,63 \\
(66.77)\end{array}$ & $\begin{array}{l}0,58 \quad-10,81 \\
(10,315.95)\end{array}$ & $\begin{array}{c}0,14 \quad 0,46 \\
(17.76)\end{array}$ & $\begin{array}{cc}0,03 & 2,28 \\
(358.35)\end{array}$ & $\begin{array}{c}0,14 \quad 0,70 \\
(37.05)\end{array}$ & $\begin{array}{cc}0,25 & 1,49 \\
(235.88)\end{array}$ & $\begin{array}{c}0,02 \quad 3,28 \\
(822.97)\end{array}$ \\
\hline $\begin{array}{c}\text { Crisis } \\
\text { asiática, } \\
\text { segunda ola }\end{array}$ & $\begin{array}{cc}0,77 & 1,02 \\
(0.54)\end{array}$ & $\begin{array}{cc}0,07 \quad 8,78 \\
(97.31)\end{array}$ & $\begin{array}{c}0,13 \quad 2,34 \\
(6.73)\end{array}$ & $\begin{array}{c}-0,02-16,17 \\
(321.10)\end{array}$ & $\begin{array}{cc}-0,02 & -37,81 \\
(1,818.24)\end{array}$ & $\begin{array}{c}-0,16-13,79 \\
(240.97)\end{array}$ & $\begin{array}{cc}-0,08 & -33,51 \\
(1,523.25)\end{array}$ & $\begin{array}{c}-0,20 \quad-6,67 \\
(56.08)\end{array}$ \\
\hline Crisis rusa & $\begin{array}{c}0,62 \quad 0,83 \\
(0.24)\end{array}$ & $\begin{array}{c}0,05 \quad 3,54 \\
(12.14)\end{array}$ & $\begin{array}{cc}-0,04 & -55,28 \\
(2,906.45)\end{array}$ & $\begin{array}{c}-0,10 \quad-7,04 \\
(47.03)\end{array}$ & $\begin{array}{c}0,08 \quad 5,95 \\
(33.50)\end{array}$ & $\begin{array}{cc}0,18 & 8,60 \\
(70.10)\end{array}$ & $\begin{array}{c}0,34 \quad 6,95 \\
(44.71)\end{array}$ & $\left.\begin{array}{ll}0,01 & 136,5 \\
(17,800.78\end{array}\right)$ \\
\hline $\begin{array}{c}\text { Crisis } \\
\text { brasileña }\end{array}$ & $\begin{array}{cc}0,33 & 0,54 \\
(0.64)\end{array}$ & $\begin{array}{cc}0,18 & 2,21 \\
(17.83)\end{array}$ & $\begin{array}{l}0,06 \quad 18,43 \\
(1,292.77)\end{array}$ & $\begin{array}{cc}0,07 & 11,17 \\
(465.48)\end{array}$ & 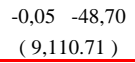 & $\begin{array}{rr}-0,01 & -358,0 \\
(468,655.59)\end{array}$ & $\begin{array}{cc}0,65 & 3,73 \\
(46.38)\end{array}$ & $\begin{array}{ll}0,02 & 87,26 \\
(28,891.44)\end{array}$ \\
\hline
\end{tabular}


Tipo de cambio

Cuadro 6

Resultados de la prueba de identificación para el tipo de cambio

\begin{tabular}{|c|c|c|c|c|c|c|c|c|}
\hline & Brasil & Rusia & Corea & Indonesia & Malasia & Filipinas & Singapur & Tailandia \\
\hline & $\beta^{1} \quad \beta^{2}$ & $\beta^{2}$ & $\beta^{1} \quad \beta^{2}$ & $\beta^{1} \quad \beta^{2}$ & $\beta^{2}$ & $\beta^{2}$ & $\beta^{2}$ & $\beta^{1}$ \\
\hline $\begin{array}{c}\text { Crisis } \\
\text { asiática, } \\
\text { primera ola }\end{array}$ & $\begin{array}{rr}-0,81 & -0,40 \\
(2,300.51)\end{array}$ & $\begin{array}{rr}0,27 & 6,19 \\
(4,006.04)\end{array}$ & $\begin{array}{l}0,04 \quad-1,83 \\
(1,342.23)\end{array}$ & $\begin{array}{c}-0,02 \quad 0,03 \\
(15.76)\end{array}$ & $\begin{array}{c}-0,06 \quad 0,28 \\
(27.54)\end{array}$ & $\begin{array}{c}0,03 \quad 0,36 \\
(6.41)\end{array}$ & $\begin{array}{c}-0,07 \quad-0,41 \\
(315.53)\end{array}$ & $\begin{array}{c}0,02 \quad-0,02 \\
(2.17)\end{array}$ \\
\hline $\begin{array}{c}\text { Crisis } \\
\text { asiática, } \\
\text { segunda ola }\end{array}$ & $\begin{array}{c}4,73 \quad 37,85 \\
(853.30)\end{array}$ & $\begin{array}{rr}-0,11 & 1323,96 \\
(868,471.53)\end{array}$ & $\begin{array}{c}0,02 \quad 3,47 \\
\quad(5.92)\end{array}$ & $\begin{array}{c}0,04 \quad 1,70 \\
(1.35)\end{array}$ & $\begin{array}{c}-0,05 \quad-12,29 \\
(76.29)\end{array}$ & 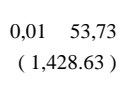 & $\begin{array}{rr}0,07 & 61,76 \\
(2,003.30)\end{array}$ & $\begin{array}{r}-0,01 \quad-54,41 \\
(1,469.89)\end{array}$ \\
\hline Crisis rusa & $\begin{array}{c}-1,52 \quad-118,10 \\
(12,017.06)\end{array}$ & $\begin{array}{c}0,02 \quad 0,41 \\
(0.14)^{*}\end{array}$ & $\begin{array}{c}0,25 \quad 6,09 \\
(31.06)\end{array}$ & $\begin{array}{c}0,03 \quad 2,17 \\
(4.03)\end{array}$ & $\begin{array}{c}0,13 \quad 8,62 \\
(62.81)\end{array}$ & $\begin{array}{c}0,09 \quad 11,91 \\
(118.75)\end{array}$ & $\begin{array}{rr}0,03 & 72,15 \\
(4,345.60)\end{array}$ & $\begin{array}{r}0,23 \quad 7,5 \\
(45.18)\end{array}$ \\
\hline $\begin{array}{c}\text { Crisis } \\
\text { brasileña }\end{array}$ & $\begin{array}{c}0,04 \quad 1,64 \\
(2.71)\end{array}$ & $\begin{array}{c}-0,06 \quad-3,00 \\
(10.12)\end{array}$ & $\begin{array}{c}0,38 \quad 10,56 \\
(120.52)\end{array}$ & $\begin{array}{c}0,12 \quad 1,38 \\
(1.82)\end{array}$ & $\begin{array}{rr}-0,21 & 154,59 \\
(24,710.21)\end{array}$ & $\begin{array}{cr}0,20 & 9,2 \\
(88.88)\end{array}$ & $\begin{array}{c}0,49 \quad 30,88 \\
(974.09)\end{array}$ & $\begin{array}{c}0,58 \quad 5,10 \\
(24.75)\end{array}$ \\
\hline
\end{tabular}

Diferenciales (Spreads) soberanos

\section{Cuadro 7}

Resultados de la prueba de identificación para los "spreads" de deuda soberana

\begin{tabular}{|c|c|c|c|c|c|c|c|c|c|c|c|c|c|c|}
\hline & \multicolumn{2}{|c|}{ Brasil } & \multicolumn{2}{|c|}{ Rusia } & \multicolumn{2}{|c|}{ Corea } & \multicolumn{2}{|c|}{ Indonesia } & \multicolumn{2}{|c|}{ Malasia } & \multicolumn{2}{|c|}{ Filipinas } & \multicolumn{2}{|c|}{ Tailandia } \\
\hline & $\beta^{1}$ & $\beta^{2}$ & $\beta^{1}$ & $\beta^{2}$ & $\beta^{1}$ & $\beta^{2}$ & $\beta^{1}$ & $\beta^{2}$ & $\beta^{1}$ & $\beta^{2}$ & $\beta^{1}$ & $\beta^{2}$ & $\beta^{1}$ & $\beta^{2}$ \\
\hline \multirow{2}{*}{$\begin{array}{c}\text { Crisis } \\
\text { asiática, } \\
\text { primera ola } \\
\text { Crisis } \\
\text { asiática, } \\
\text { segunda ola }\end{array}$} & ( 5.80$)$ & $\begin{array}{l}2,12 \\
0)\end{array}$ & $\begin{array}{r}-0,01 \\
(\end{array}$ & $\begin{array}{l}0,14 \\
2)\end{array}$ & ( 735.12$)$ & $\begin{array}{l}9,21 \\
12)\end{array}$ & $\begin{array}{l}0,04 \\
(23,\end{array}$ & $\begin{array}{c}-78,65 \\
31.17 \text { ) }\end{array}$ & $\begin{array}{r}0,56 \\
\quad(11\end{array}$ & $\begin{array}{l}-6,35 \\
.81)\end{array}$ & $\begin{array}{r}1,32 \\
(2\end{array}$ & $\begin{array}{l}2,35 \\
02)\end{array}$ & $\begin{array}{r}-1,61 \\
(32\end{array}$ & $\begin{array}{l}-6,53 \\
.72)\end{array}$ \\
\hline & $\begin{array}{r}0,82 \\
\quad(0\end{array}$ & $\begin{array}{l}1,21 \\
)^{*}\end{array}$ & 0,01 & $\begin{array}{l}0,75 \\
)^{*}\end{array}$ & $\begin{array}{r}0,39 \\
\quad(1\end{array}$ & $\begin{array}{l}3,29 \\
)^{*}\end{array}$ & $\begin{array}{r}0,37 \\
\quad(1\end{array}$ & $\begin{array}{l}7,69 \\
.69)\end{array}$ & $\begin{array}{r}-31,28 \\
(1,0\end{array}$ & $\begin{array}{c}14,12 \\
1.51)\end{array}$ & & $\begin{array}{l}3,37 \\
0)\end{array}$ & $\begin{array}{r}-0,20 \\
(9\end{array}$ & $\begin{array}{l}-7,17 \\
72)\end{array}$ \\
\hline Crisis rusa & \multicolumn{2}{|c|}{$(0.01)^{*}$} & \multicolumn{2}{|c|}{$(0.00)^{*}$} & $(3.22)^{*}$ & $\begin{array}{l}9,78 \\
)^{*}\end{array}$ & & $\begin{array}{l}3,31 \\
3)^{*}\end{array}$ & $(17.79)$ & $\begin{array}{l}-24,34 \\
.79)\end{array}$ & $(0.28)^{*}$ & $\begin{array}{l}3,70 \\
3 *\end{array}$ & $\begin{array}{r}-0,15 \\
(2\end{array}$ & $\begin{array}{l}-25,8 \\
74)\end{array}$ \\
\hline $\begin{array}{c}\text { Crisis } \\
\text { brasileña }\end{array}$ & $\begin{array}{r}0,52 \\
\quad(0\end{array}$ & $\begin{array}{l}0,80 \\
)^{*}\end{array}$ & $\begin{array}{l}0,00 \\
(C\end{array}$ & $\begin{array}{l}-0,29 \\
)^{*}\end{array}$ & $\begin{array}{r}1,22 \\
(3\end{array}$ & $\begin{array}{l}6,50 \\
*\end{array}$ & $\begin{array}{r}0,44 \\
(4\end{array}$ & $\begin{array}{l}6,79 \\
01)\end{array}$ & $\begin{array}{l}0,41 \\
\quad(16\end{array}$ & $\begin{array}{c}13,94 \\
.55)\end{array}$ & $\begin{array}{r}2,42 \\
\quad(3\end{array}$ & (1) & $\begin{array}{r}1,62 \\
\quad(3\end{array}$ & $\begin{array}{l}19,40 \\
37 \text { ) }\end{array}$ \\
\hline
\end{tabular}

Se observan muy pocas diferencias significativas entre los coeficientes, lo cual indica que solamente hay evidencia de contagio en los mercados de tipo de cambio en el caso de la crisis rusa y en la segunda ola de la crisis asiática. También existe evidencia de contagio en el mercado de deuda soberana sólo durante la crisis rusa, la crisis brasileña y la segunda ola de la crisis asiática, particularmente en el caso de Corea.

Los resultados presentados en esta sección documentan la presencia de contagio de las crisis financieras recientes hacia México, a partir de la segunda ola de la crisis asiática. Sin embargo, al parecer el contagio se intensificó realmente después de la crisis rusa. Una de las principales causas de ello fue la asociación, en las mentes de los inversionistas, de los desarrollos económicos en Brasil y México con lo sucedido en Rusia. 


\section{Canales de Transmisión y la Respuesta de Política}

Siguiendo el trabajo realizado por Masson (1998) es posible esgrimir muchas razones por las que las crisis en distintos países pueden ser contemporáneas. En primer lugar, estas crisis pueden deberse a factores comunes, como el incremento en las tasas de interés mundiales o la caída en los precios mundiales de los bienes básicos de exportación. Otra posibilidad, subrayada por Corsetti et al (1998), es que la crisis en un país deteriore los fundamentos económicos de otro país, llevándolo a condiciones de mayor volatilidad. Finalmente, Masson señala que una crisis puede disparar otra crisis en otra nación por razones no explicables a través de los fundamentos económicos, tal vez porque genera un cambio en el "sentimiento" del mercado. Este último tipo de episodio es descrito por Masson como "contagio verdadero". Técnicamente, Masson relaciona este "contagio verdadero" con una situación de equilibrios múltiples donde las noticias de una crisis en un país disparan un movimiento de un equilibrio estable y "bueno" hacia un equilibrio inestable o "malo" en otra nación.

Como se mencionó en la introducción, la crisis de Asia impactó a las economías latinoamericanas primero a través de su efecto sobre los precios de las materias primas, dañando las exportaciones y las finanzas públicas en la región. Además, aquellas naciones que mantienen lazos comerciales cercanos con Asia, o que compiten con las exportaciones asiáticas en terceros países, fueron afectadas negativamente por la contracción de la demanda asiática y por la depreciación de las monedas de estos países. En el caso de México, la economía experimentó un deterioro marcado en sus términos de intercambio durante 1998 (de 5.5\%), debido en su mayor parte a la caída en el precio del petróleo. Al principio de la crisis se pensó que, aunque la proporción del comercio exterior de México que se realiza con los países en crisis era extremadamente pequeña, los lazos indirectos con los países asiáticos, a través de la competencia con las exportaciones mexicanas en el mercado americano, no eran despreciables. En 1998, un estudio realizado por el Banco de México llegó a la conclusión de que tal vez haya una competencia muy intensa de los productos asiáticos en los mercados de Estados Unidos en aquellas líneas de producción en las que las exportaciones Mexicanas constituyen menos que el 10\% de las importaciones de EE.UU.. Sin embargo, en aquellos bienes en que las exportaciones mexicanas representan más de $10 \%$ de las importaciones de EE.UU., la competencia de los países asiáticos es baja o moderada ${ }^{4}$. Para cuantificar, ex-post, el grado de sustitución, se observó si en los 1250 artículos importados por los Estados Unidos, la participación de las exportaciones mexicanas y de otros países no asiáticos, en las importaciones de EE.UU., disminuyó y si, obviamente, la participación de las exportaciones asiáticas aumentó. De ocurrir lo anterior, se considera que hubo sustitución. Este cálculo señala que durante 1998, 1.3 billones de dólares de exportaciones mexicanas (1.1\% de las exportaciones totales) fueron desplazadas por exportaciones de Asia. A pesar de este fenómeno, México fue capaz

4 Ver “The Mexican Economy” para 1998 y 1999, publicado por Banco de México. 
de aumentar su participación en las importaciones totales de los Estados Unidos a través de incrementos en otras exportaciones. Por tanto, el contagio a través de este canal fue muy reducido.

\section{Gráfica 5}

Exportaciones de México a los EE.UU. como proporción del total de importaciones de este último país

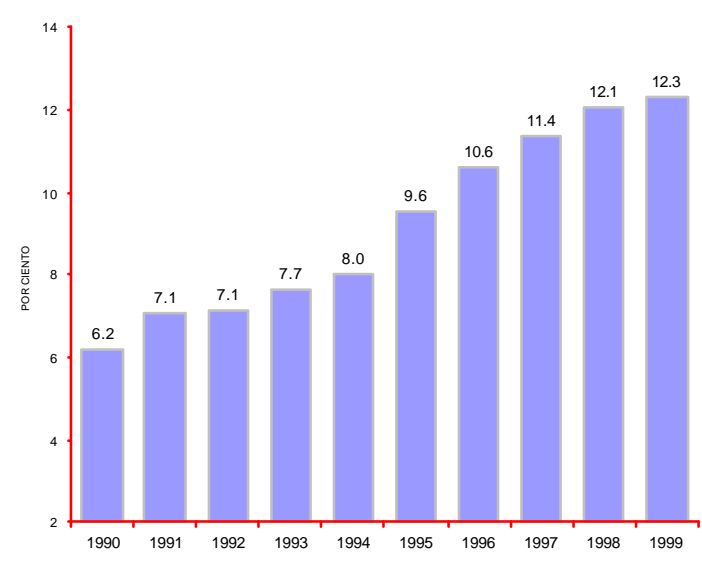

Total de las exportaciones no petroleras mexicanas

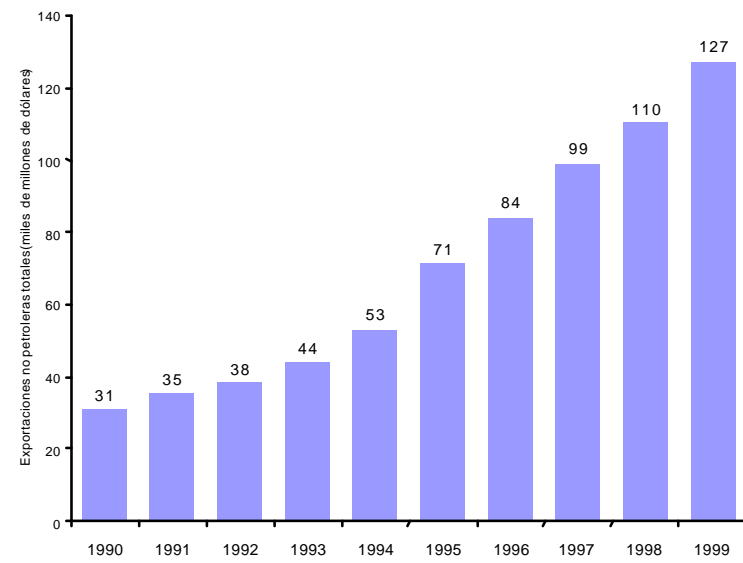

Aun cuando el impacto en las cuentas externas fue despreciable, la caída en los precios del petróleo afectó significativamente los ingresos públicos, dado que $36.4 \%$ de los ingresos del gobierno se obtuvieron del petróleo durante 1997. Por tanto, en este caso, la crisis de Asia, a través de su impacto en el precio del petróleo sí afectó los fundamentos económicos de México. La pérdida de ingresos públicos fue por un total de $1.3 \%$ del PIB.

La política fiscal reaccionó de inmediato ante la pérdida de ingresos derivada de la caída en el precio del petróleo ( de un precio previsto de $15.5 \mathrm{dpb}$ a un nivel de $10.16 \mathrm{dpb}$ ). Durante 1998, se aplicaron tres ajustes al presupuesto y se llevó a cabo un importante esfuerzo para reducir la evasión de impuestos. Estas medidas compensaron completamente la caída en los ingresos públicos, e hicieron posible alcanzar un déficit de $1.24 \%$ del PIB, ligeramente inferior al programado a principios del año. Se debe señalar que este esfuerzo fiscal se realizó en adición a las medidas fiscales tomadas durante los últimos cuatro años para financiar los costos de la reforma al sistema de pensiones y los diversos programas encaminados hacia la reestructuración del sistema financiero. El ajuste fiscal resultó ser de extrema importancia, dado que durante la segunda mitad de 1998, el financiamiento internacional se colapsó, y, consecuentemente, hubiera sido extremadamente costoso financiar un mayor déficit del sector público.

La respuesta fiscal, y el reducido impacto de la crisis asiática sobre las cuentas externas de México minimizó el efecto de la crisis en la economía. Sin embargo, durante agosto, el colapso del rublo ruso y la moratoria de la deuda de ese país tuvo consecuencias serias sobre las economías emergentes. Esto se debió a las siguientes razones: 
a) dado que importantes cantidades de recursos de fondos de inversión estaban atrapadas en Rusia, estos fondos se vieron obligados a liquidar sus posiciones en otros mercados más líquidos, como el de México.

b) en ausencia de un apoyo coordinado del Fondo Monetario Internacional y los países del G7 a Rusia, el falso sentimiento de seguridad, basado en la noción de que algunos países eran "demasiado grandes para caer" se evaporó. Lo anterior dio lugar a una importante revaluación del riesgo en mercados emergentes, generándose así agudos problemas de refinanciamiento en estos mercados, y

c) debido al cambio en la percepción del riesgo en mercados emergentes, los inversionistas empezaron a anticipar la devaluación de la moneda brasileña. Bajo el supuesto de que el peso estaba altamente correlacionado con el real brasileño, los futuros del peso se volvieron una vía útil para cubrir el riesgo brasileño. Así, el tipo de cambio del peso se vio afectado, puesto que las instituciones bancarias mexicanas, la contraparte de las operaciones de cobertura mencionadas anteriormente, equilibraron sus posiciones comprando dólares a cambio de pesos en el mercado "spot". 5

La Gráfica 6 muestra claramente la relación inversa entre la posición no cubierta en instrumentos de renta fija en moneda nacional y el valor del Peso contra el Dólar.

\section{Gráfica 6}

Posición no cubierta en instrumentos de renta fija y el tipo de cambio peso/dólar

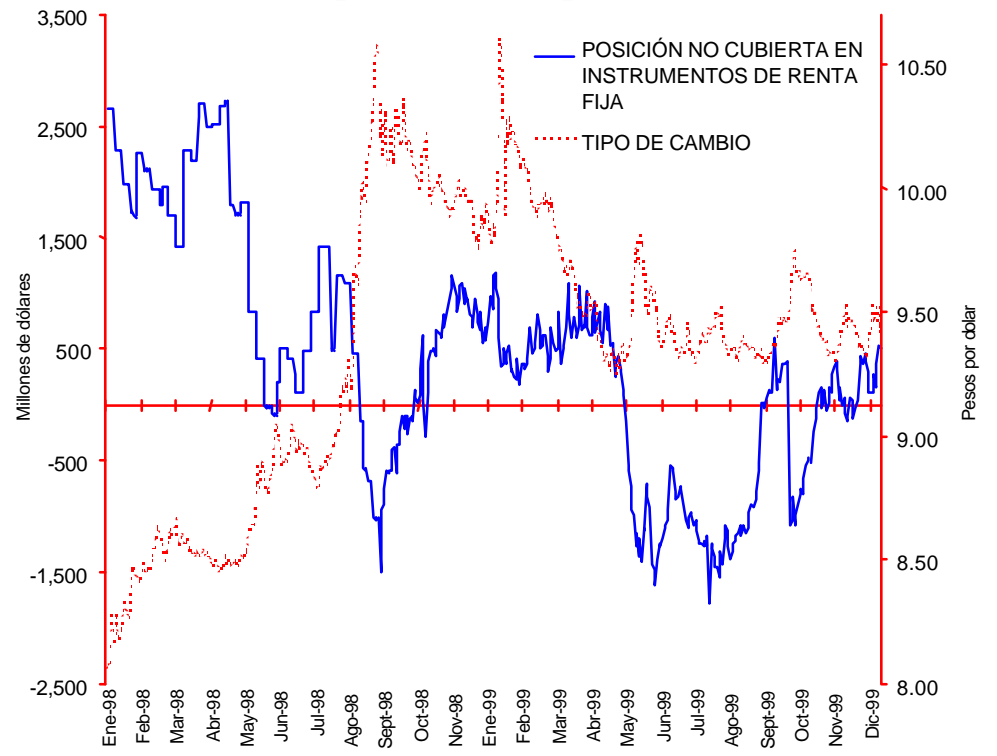

5 Este problema se analiza también en Kodres y Pritsker (1998) y en Jochum y Kodres (1998). 
Cuando la posición no cubierta se vuelve negativa, los inversionistas extranjeros están vendiendo pesos en los mercados de futuros en cantidades superiores a sus acervos de instrumentos denominados en pesos. Para validar esta cadena de implicaciones, se estimó un modelo VAR, con datos diarios para un período de un año, empezando dos semanas antes de la devaluación y moratoria rusa ${ }^{6}$, en el cual se incluyen las variables de los "spreads" soberanos de Rusia (RUSIA), los de Brasil (BRASIL), los de México (MÉXICO), la posición no cubierta de extranjeros en pesos en México (PNC) y el tipo de cambio peso/dólar (TC). Las funciones de impulso respuesta, presentadas en la Gráfica 7, justifican el canal de contagio descrito anteriormente. Las perturbaciones al riesgo país de Rusia no afectaron de manera importante el de Brasil ni el de México. Sin embargo, una perturbación directa al riesgo país de Brasil tuvo un fuerte impacto en el de México, así como en las ventas de futuros del peso y, por tanto, generó una depreciación de la moneda. De la estimación del VAR es claro que las variables de México responden básicamente a la perturbación observada en Brasil y no a la ocurrida en Rusia.

6 Se realizó el mismo ejercicio para un periodo que no incluyese la crisis brasileña, de agosto 13 de 1998 hasta enero8, 1999 obteniendo resultados muy similares en términos de las funciones de impulso respuesta y la descomposición de varianza. 


\section{Gráfica 7}

Funciones de impulso respuesta de la estimación VAR
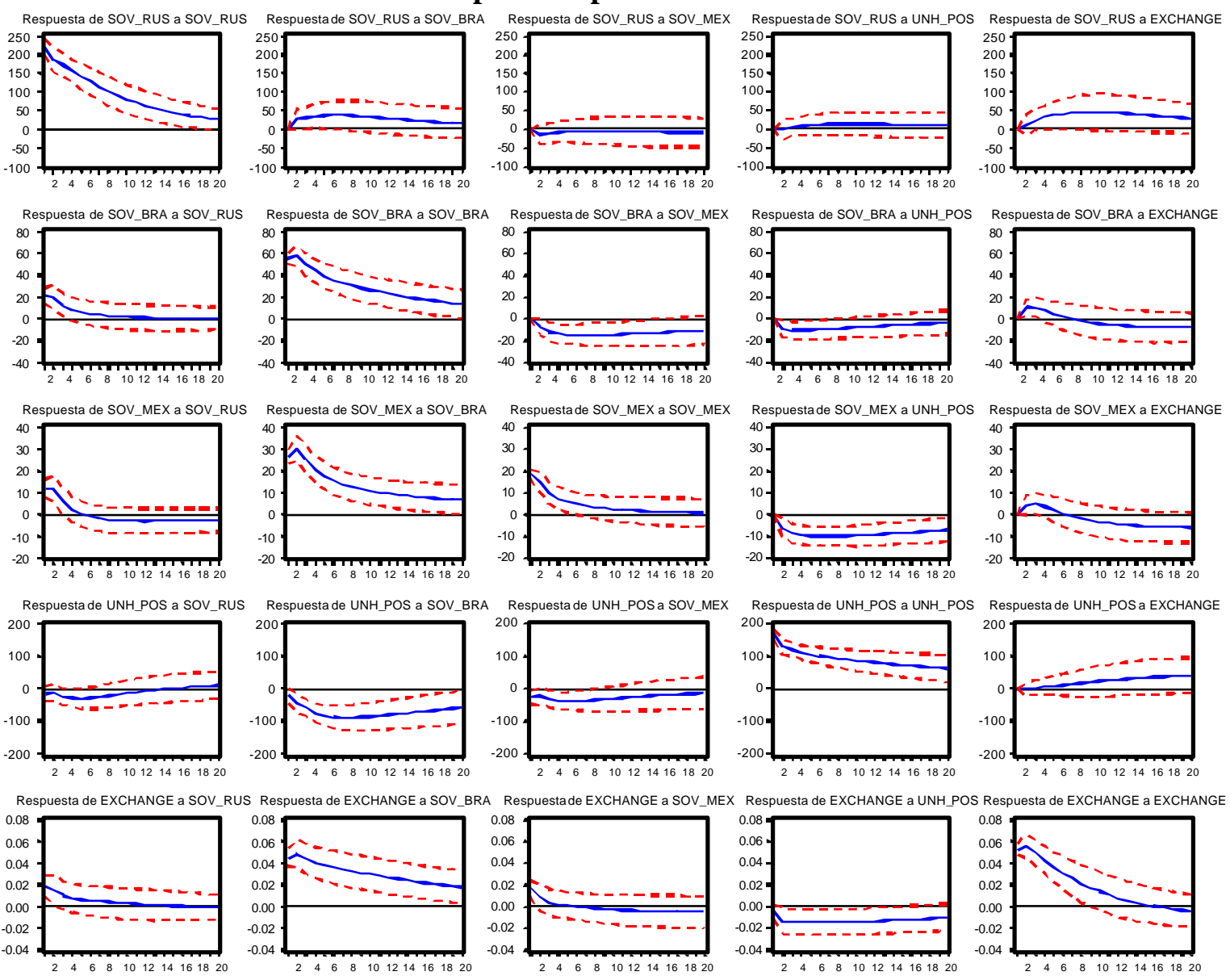

La descomposición de varianza del VAR anterior muestra que durante este periodo la varianza de los "spreads" mexicanos provino en gran medida de Brasil, y que Rusia no contribuye mucho a esta varianza. En cuanto a la posición no cubierta y el tipo de cambio, la proporción de la varianza explicada por Brasil también es importante, de alrededor de $30 \%$ y $50 \%$ respectivamente, después de seis semanas. Por tanto, aún cuando las perturbaciones en Rusia aparentemente tuvieron un efecto inmediato y transitorio en México, posiblemente éste no hubiera existido si no fuera por la transmisión a través de los mercados de Brasil, los cuales afectaron considerablemente el comportamiento de los mercados nacionales. 


\section{Cuadro 9}

Descomposición de la varianza de los choques

en la especificación VAR

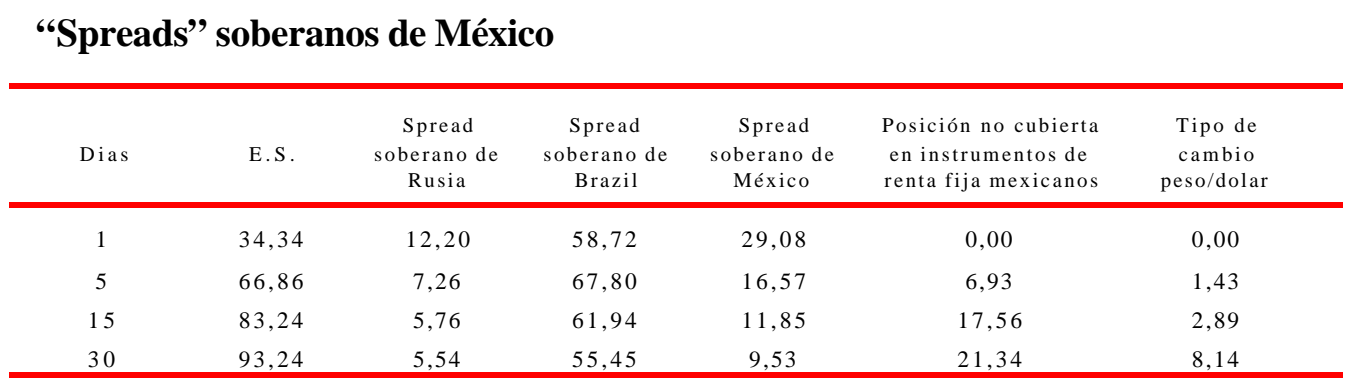

Posición no cubierta en instrumentos de renta fija mexicanos

\begin{tabular}{ccccccc}
\hline Dias & E.S. & $\begin{array}{c}\text { Spread } \\
\text { soberanode } \\
\text { Rusia }\end{array}$ & $\begin{array}{c}\text { Spread } \\
\text { soberanode } \\
\text { Brazil }\end{array}$ & $\begin{array}{c}\text { Spread } \\
\text { soberanode } \\
\text { México }\end{array}$ & $\begin{array}{c}\text { Posición no cubierta } \\
\text { en instrumentos de } \\
\text { renta fija mexicanos }\end{array}$ & $\begin{array}{c}\text { Tipo de } \\
\text { cambio } \\
\text { peso/dolar }\end{array}$ \\
\hline 1 & 170,44 & 0,90 & 1,58 & 2,67 & 94,85 & 0,00 \\
5 & 327,72 & 2,62 & 17,10 & 4,73 & 75,45 & 0,10 \\
15 & 517,91 & 2,08 & 32,85 & 5,37 & 57,43 & 2,27 \\
30 & 621,93 & 1,85 & 33,76 & 4,33 & 52,28 & 7,78 \\
\hline
\end{tabular}

\section{Tipo de cambio peso/dólar}

\begin{tabular}{ccccccc}
\hline Dias & E.S. & $\begin{array}{c}\text { Spread } \\
\text { soberano de } \\
\text { Rusia }\end{array}$ & $\begin{array}{c}\text { Spread } \\
\text { soberanode } \\
\text { Brazil }\end{array}$ & $\begin{array}{c}\text { Spread } \\
\text { soberanode } \\
\text { México }\end{array}$ & $\begin{array}{c}\text { Posición } \\
\text { en instrumentos de } \\
\text { rentafijamexicanos }\end{array}$ & $\begin{array}{c}\text { Tipo de } \\
\text { cambio } \\
\text { peso/dolar }\end{array}$ \\
\hline 1 & 0,07 & 6,59 & 37,24 & 5,08 & 0,42 & 50,67 \\
5 & 0,15 & 3,43 & 41,08 & 1,58 & 3,68 & 50,23 \\
15 & 0,19 & 2,39 & 49,68 & 1,24 & 7,46 & 39,22 \\
30 & 0,21 & 2,09 & 51,86 & 1,82 & 9,36 & 34,87 \\
\hline
\end{tabular}

La moratoria rusa y la intensificación de las presiones especulativas en Brasil llevaron a una severa contracción de los flujos de capital hacia los mercados emergentes, lo cual se reflejó en un aumento significativo de los "spreads" soberanos de la deuda de las economías emergentes.

La caída en los términos de intercambio de México y más aún, el colapso de los mercados internacionales de capital llevaron a una importante depreciación del tipo de cambio. Dada la apertura de la economía mexicana, y la alta correlación histórica entre la depreciación del tipo de cambio y la inflación, estos movimientos cambiarios tuvieron un efecto inflacionario significativo. Más aún, una proporción importante del efecto de la caída del precio del petróleo sobre las finanzas públicas se compensó vía aumentos en los precios de los bienes y servicios suministrados por el sector público, complicando aún más la perspectiva inflacionaria. La autoridad monetaria reaccionó a estas presiones endureciendo progresivamente la política monetaria, de tal forma que las tasas de interés de corto plazo pasaron de un mínimo de $17.75 \%$ en mayo de 1998 hasta un máximo de $41.33 \%$ en el punto más álgido de la crisis. 


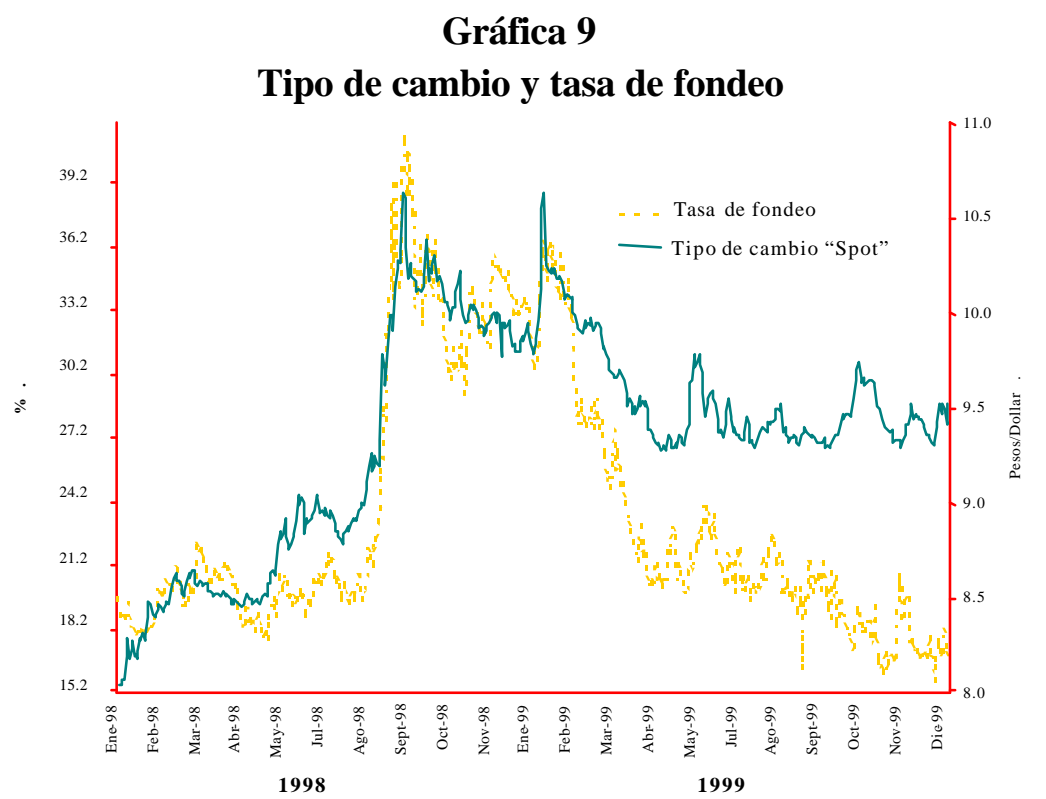

A pesar de la crisis, durante 1998-1999 la economía mexicana mostró un comportamiento favorable, más aún si se compara con el de otras economías, tanto desarrolladas como emergentes. Así, el PIB creció 4.8 y $3.7 \%$, el empleo aumentó 7.8 y $6.1 \%{ }^{7}$, las exportaciones subieron 11.3 y $14.9 \%$ y las reservas internacionales se incrementaron. Sin embargo, dados los impactos inflacionarios descritos anteriormente la tasa de inflación se desvió 6.6 puntos porcentuales de su objetivo original de $12 \%$ en 1998 y en 1999 se cumplió con el objetivo de obtener una inflación menor que $13 \%$.

Incremento porcentual en el número de trabajadores afiliados al Instituto Mexicano del Seguro Social. 


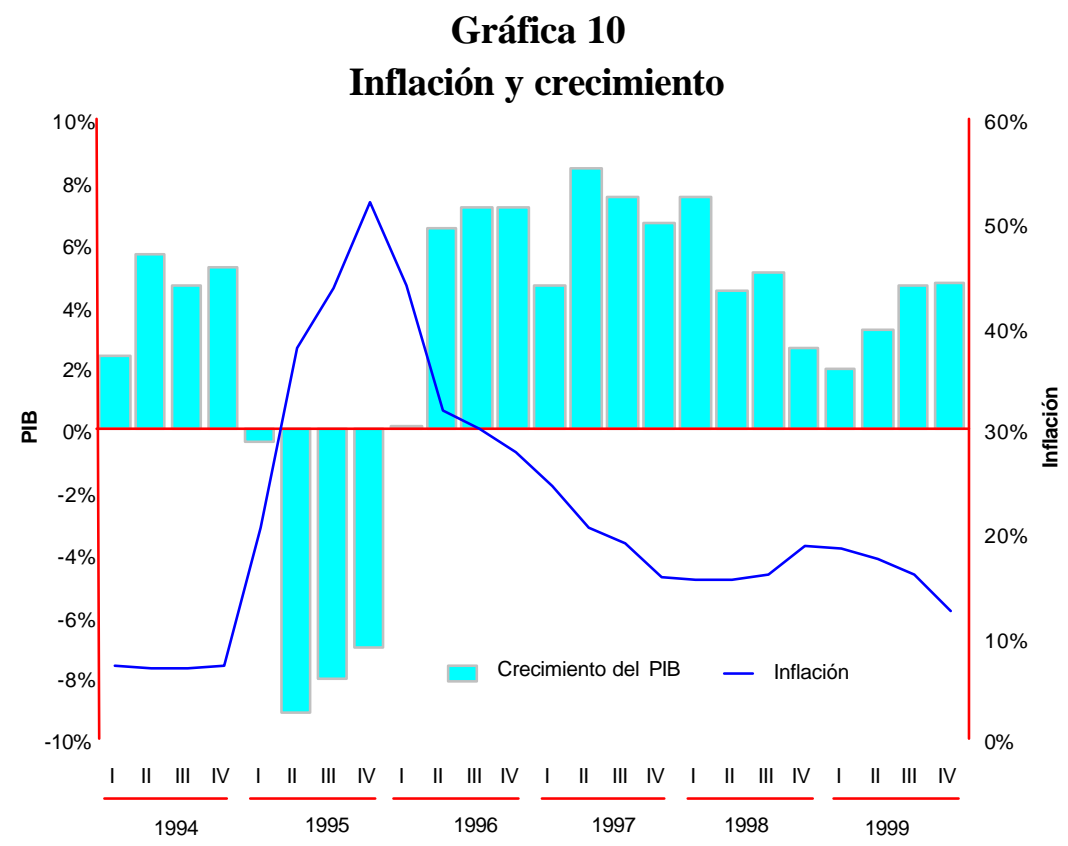

Además de la rápida reacción del sector público, y de los efectos positivos del dinamismo de la economía americana, el régimen de tipo de cambio flexible facilitó el ajuste del tipo de cambio real hacia un nuevo equilibrio tras las perturbaciones externas sin que se erosionara seriamente la credibilidad de la autoridad monetaria.

El mejor ejemplo de esta función que juega el tipo de cambio es lo que se pudo observar durante 1998 con las monedas de Australia, Canadá y Nueva Zelandia. Estas economías pequeñas y abiertas fueron severamente afectadas por el deterioro de los términos de intercambio de sus economías y por la caída en la demanda de los países asiáticos. Como una reacción a estas perturbaciones las monedas de estos países sufrieron depreciaciones importantes. Estos movimientos en el valor de sus monedas sirvieron para mitigar el efecto real de las perturbaciones antes mencionadas. Un fenómeno similar se dio en México, al caer los términos de intercambio del país en $5.5 \%$ y cerrarse las puertas de los mercados financieros internacionales para las economías emergentes. En teoría ambas perturbaciones deben depreciar el tipo de cambio real de equilibrio, justificando la depreciación nominal observada. 


\section{Gráfica 11 \\ Tipo de cambio real (1998)}

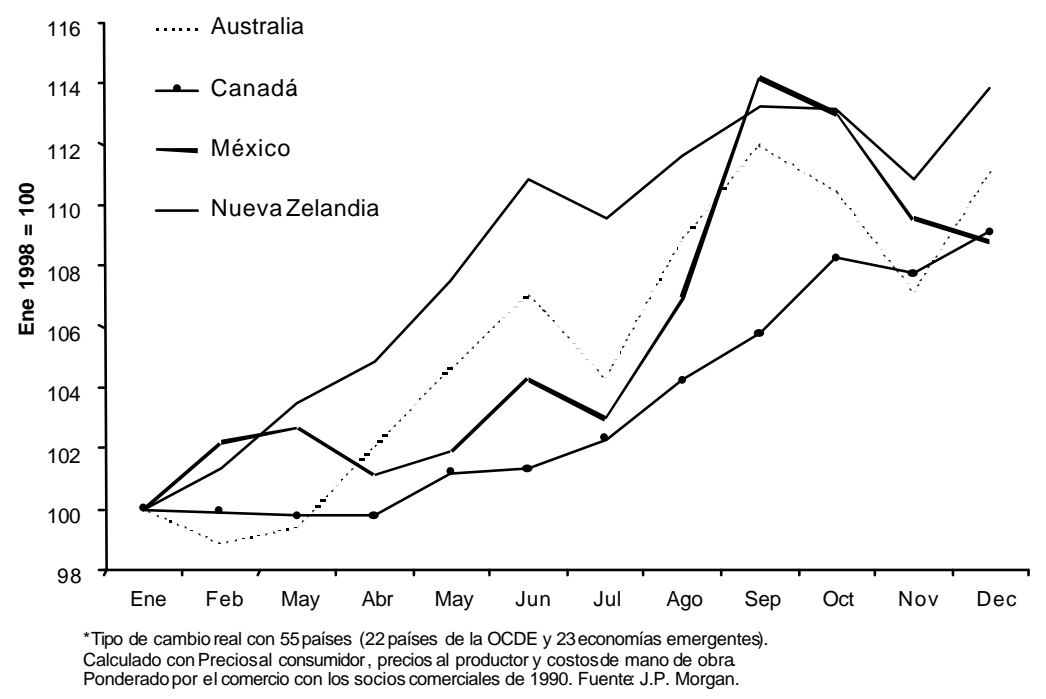

Para evaluar el impacto de estas depreciaciones del tipo de cambio sobre la actividad, se puede comparar el comportamiento de estas economías pequeñas abiertas con regímenes de tipo de cambio flexible, con el de países con esquemas de tipo de cambio extremadamente fijos, como Argentina y Hong Kong.

Cuadro 11

Crecimiento del PIB en 1998 - 1999

\begin{tabular}{lrc}
\hline & 1998 & 1999 \\
\hline Argentina & 3.9 & -2.7 \\
Hong Kong & -5.1 & 0.6 \\
México & 4.8 & 3.7 \\
Canadá & 3.1 & 3.7 \\
Australia & 4.8 & 4.8 \\
Nueva Zelandia & 0.3 & 3.0 \\
\hline
\end{tabular}

Datos: Goldman Sachs, J.P. Morgan, Enero 2000

Sin embargo, dado que México tiene una historia de alta inflación y considerando que históricamente se observa una alta correlación ente la inflación y la depreciación del tipo de cambio, las expectativas de inflación se deterioraron de inmediato. Por tanto, el costo inflacionario de lograr la corrección en el tipo de cambio real fue de alrededor de 2.2 puntos porcentuales. Por otra parte, la depreciación nominal necesaria para lograr el mismo movimiento en el tipo de cambio real tuvo que ser significativamente más grande que las observadas en los demás países considerados. 


\section{Gráfica 12}

\section{Tasa de inflación y depreciación del peso}

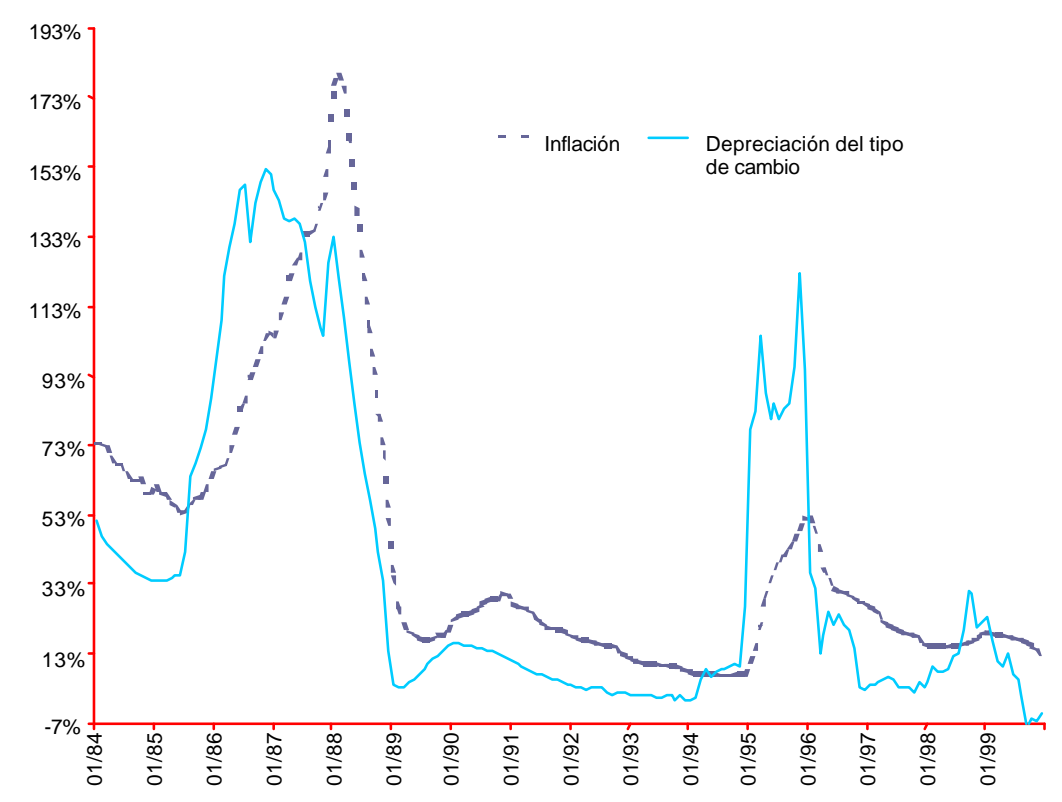

Una comparación de la velocidad del passthrough en México y en Australia señala el problema que tiene México debido a su historia de alta inflación. Aun cuando el efecto de largo plazo de una depreciación del tipo de cambio en los precios no controlados es del mismo orden de magnitud en México y en Australia (una depreciación de $1 \%$ se traduce en inflación de $0.66 \%$ en México y de $0.44 \%$ en Australia), en México la mitad de este efecto se da después de dos trimestres, y $82 \%$ se manifiesta antes de un año. En contraste, en Australia, solo 7\% del efecto tiene lugar en dos trimestres y sólo $14 \%$ en un año ${ }^{8}$.

Reducir la velocidad con la que las depreciaciones del peso se convierten en inflación, es uno de los principales retos que la política monetaria enfrenta en el futuro. Ello, con el fin de que México pueda aprovechar las ventajas de régimen de libre flotación y que el tipo de cambio juegue el papel de un precio relativo más en la economía, en lugar de ser una señal sobre el comportamiento de la inflación futura.

Otro factor importante que contribuyó a reducir el impacto de la crisis financiera internacional en la economía mexicana es que el país ha logrado fortalecer sus fuentes de financiamiento exentas de volatilidad. En contraste con lo sucedido en 1994, para los años de 1997-1998, casi el 80\% del financiamiento de la cuenta corriente provino de entradas de capital de largo plazo, IED en particular. Adicionalmente, las transferencias hechas por los mexicanos establecidos en los Estados Unidos alcanzaron casi 6 mil millones de dólares. Hacia el final de 1997, el gobierno negoció con bancos extranjeros una línea de crédito por 1.69 miles de millones de dólares para usarse en caso de una contingencia adversa. El gobierno aprovechó estos recursos en septiembre de 1998.

8 Esta comparación se realiza en el trabajo de Garcés (1999). 
Finalmente, ciertas corporaciones privadas fueron capaces de obtener financiamiento extranjero a partir de créditos con la banca comercial, o de sus proveedores y, en el caso de las compañías filiales, de las casas matrices en el extranjero.

\section{Gráfica 13}

\section{Déficit en cuenta corriente e inversión extranjera directa como porcentaje del PIB}

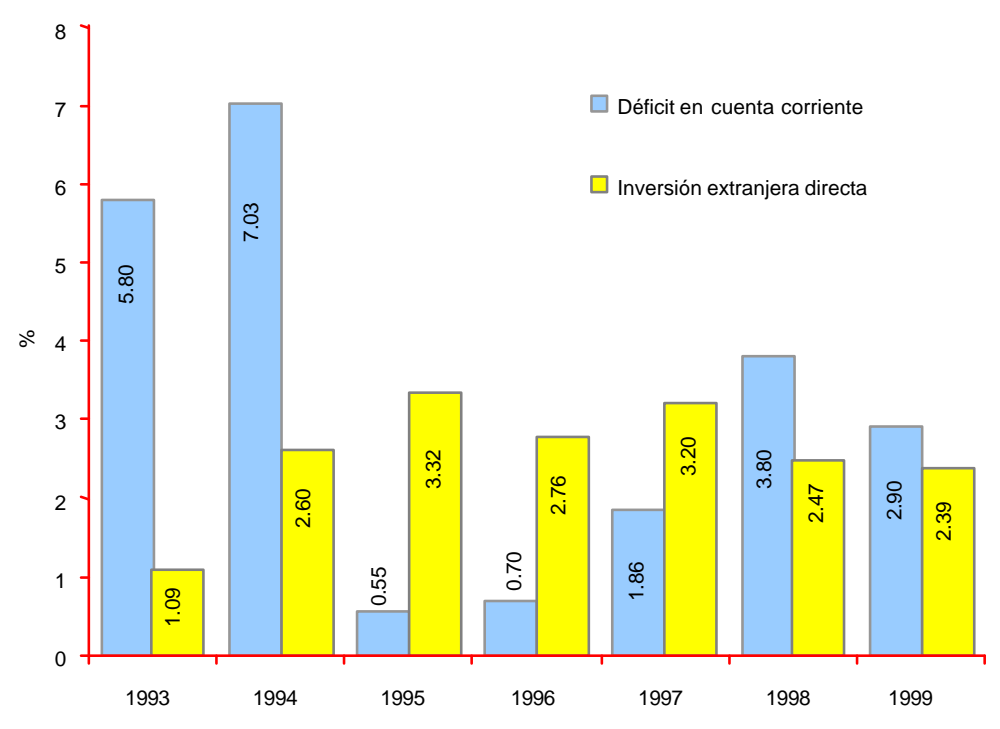

\section{Conclusiones}

En este documento se estudió el contagio sufrido por los mercados financieros de México, de la reciente crisis financiera global. Utilizando varias metodologías, encontramos que hubo contagio hacia México, particularmente después de la segunda ola de la crisis asiática, y que éste se intensificó tras la declaración de la moratoria de pagos de Rusia.

Sin embargo, la economía mexicana sufrió relativamente poco de este contagio de sus mercados financieros pues estaba en una posición sólida para contener estos choques. A este respecto es importante subrayar el efecto positivo que el dinamismo de la economía americana ha tenido sobre la evolución económica de México. Además, las políticas fiscal y monetaria conservadoras implementadas durante el período, así como la posición fuerte de liquidez y el bajo déficit en cuenta corriente, redujeron las fuentes de vulnerabilidad de la economía a los choques externos. 


\section{Bibliografía}

T. Baig y I. Goldfajn (1998) "Financial Markets Contagion in the Asian Crisis," Working Paper 98/155, Fondo Monetario Internacional.

Banco de México, The Mexican Economy, Varios números, México D.F.

S. Calvo y C. Reinhart (1996), "Capital Flows to Latin America: Is there Evidence of Contagion Effects?" en Private Capital Flows to Emerging Markets after the Méxican Crisis, Calvo, Goldstein, Hochreiter (eds).

G. Corsetti, P. Pesenti y N. Roubini (1998), "What Caused the Asian Currency and Financial Crisis," NBER Working Paper No. 6833,6834.

K. Forbes y R. Rigobon, (1999), "No Contagion, Only Interdependence: Measuring Stock Market Co-movements," NBER Working Paper No. 7267

D. Garcés, (1999), "Determinación del nivel de precios y la dinámica inflacionaria en México", Mimeo, Banco de México, Diciembre.

J. Hamilton, (1994), Time Series analysis, Princeton University Press, New Jersey.

Ch. Jochum y L. Kodres, (1998), "Does the Introduction of Futures on Emerging Market Currencies Destabilize the Underlying Currencies?", IMF Working Paper No. 98/13, Fondo Monetario Internacional, Febrero.

L. Kodres y M. Pritsker, (1998), “A rational Expectations Modelo of Financial Contagion”, Banco de la Reserva Federal, Serie de discusión \# 1998-48, Noviembre.

P. Masson, (1998), 'Contagion: Monsoonal Effects, Spillovers, and Jumps Between Multiple Equilibria," IMF Working Paper No. 98/142-EA. , Fondo Monetario Internacional, Septiembre.

R. Rigobon, (1999), 'On the Measurement of the International Propagation of Schocks", NBER Working Paper No. 7354 , Septiembre.

R. Rigobon, (1999), 'Identification through Heteroskedasticity: Measuring "Contagion” between Argentinean and Mexican Sovereign Bonds," mimeo de MIT, Diciembre.

J. Sachs, A. Tornell y A. Velasco, (1996), 'Financial Crisis in Emerging Markets: The Lessons from 1995," NBER Working Paper No. 5576, Mayo.

R. Valdés, (1997), "Emerging Markets Contagion: Evidence and Theory,” Documentos de Trabajo del Banco Central, Banco central de Chile. 